\title{
Quantum Chemical Calculations
}

DFT calculations of various scalar couplings $\left({ }^{3} J_{\mathrm{HN}, \mathrm{C}},{ }^{3} J_{\mathrm{HN}, \mathrm{C} \beta}\right.$ and $\left.{ }^{3} J_{\mathrm{HN}, \mathrm{H \alpha}}\right)$ were carried out on Ace-Xaa-NMe dipeptide analogues $(\mathrm{Xaa}=$ Ala or the other amino acid residues listed in Table S1) using Gaussian0 $0{ }^{1}$ running on the Biowulf cluster at the Division of Computer Research and Technology at the National Institutes of Health. In all calculations, ideal local covalent geometries were initially generated with the $(\phi$ and $\psi)$ backbone angles set to $\left(-60^{\circ}\right.$ and $\left.-45^{\circ}\right)$ for a "helix" structure and to $\left(-120^{\circ}\right.$ and $\left.120^{\circ}\right)$ for a "sheet" structure. After partial geometry optimization using the B3LYP hybrid functional ${ }^{2}$ and the $6-311 \mathrm{G}^{* *}$ basis set, the coupling constants including the Fermi contact (FC), spin-dipolar (SD), paramagnetic spin-orbit (PSO), and diamagnetic spinorbit (DSO) contributions ${ }^{3,4}$ were then calculated on the resulting geometries at the same level of theory.

Residue type and $\chi_{1}$ effect on ${ }^{3} J$. The effect of amino acid residue type and sidechain $\chi_{1}$ rotameric state was investigated by replacing the Ala residue Ace-Ala-NMe. In all cases, the $\chi_{1}$ torsion angles were set to $60^{\circ}$ for the gauche+, $-60^{\circ}$ for the gauche-, and $180^{\circ}$ for the trans configuration in the initial geometry. Values for $\chi_{2}$ and other side chain torsion angles further removed from the backbone were set to a value found for a particular residue type in the crystal structure of Protein G (PDB code: 1IGD). Except for Asp ${ }^{-}$and $\mathrm{Glu}^{-}$, none of the side chain torsion angles were constrained in the optimization, which resulted in only small deviations $\left(<20^{\circ}\right)$ from the ideal $\chi_{1}$ values in the optimized geometry. For $\mathrm{Asp}^{-}$and $\mathrm{Glu}^{-}$, due to the interactions between the negatively charged side chain and the amide group, geometry optimization resulted in much larger deviations from the starting, ideally staggered rotamers, and explicit restraints retaining the staggered $\chi_{1}$ rotamers were used to constrain these angles. To eliminate the effect of variations in bond angles and dihedral angles on couplings to be calculated, all bond angles involving the amide $\mathrm{N}$ and the $\mathrm{C}^{\alpha}$ atoms were fixed during the partial geometry optimization, and the $\phi$ and $\psi$ backbone angles were frozen during the optimization. Improper angles were constrained to maintain peptide planarity during the partial geometry optimization. Calculation of the scalar coupling constants then was performed using the optimized geometry, resulting in the couplings given in Table S1. 
Table S1. Calculated ${ }^{3} J$ couplings for various residue types.

\begin{tabular}{|c|c|c|c|c|c|c|}
\hline \multirow{2}{*}{ residue, $\chi_{1}$ angle } & \multicolumn{3}{|c|}{ couplings for $\alpha$-Helix $(\mathrm{Hz})$} & \multicolumn{3}{|c|}{ couplings for $\beta$-Sheet $(\mathrm{Hz})$} \\
\hline & ${ }^{3} J_{\mathrm{HN}-\mathrm{C}^{\prime}}$ & ${ }^{3} J_{\mathrm{HN}, \mathrm{C} \beta}$ & ${ }^{3} J_{\mathrm{HN}, \mathrm{H} \alpha}$ & ${ }^{3} J_{\mathrm{HN}, \mathrm{C}^{\prime}}$ & ${ }^{3} J_{\mathrm{HN}, \mathrm{C} \beta}$ & ${ }^{3} J_{\mathrm{HN}, \mathrm{H} \alpha}$ \\
\hline Ala & 1.59 & 3.93 & 2.31 & 0.30 & 0.77 & 9.65 \\
\hline Asn, $g+$ & 1.50 & 3.57 & 2.16 & 0.38 & 0.39 & 9.68 \\
\hline Asn, $g-$ & 1.45 & 3.64 & 2.40 & 0.25 & 0.69 & 9.65 \\
\hline Asn, $t$ & 1.54 & 3.63 & 2.41 & 0.36 & 0.34 & 10.25 \\
\hline Asp, $g+$ & 1.56 & 2.76 & 2.82 & 0.52 & 0.39 & 9.76 \\
\hline Asp, $g$ - & 1.97 & 2.86 & 2.56 & 0.04 & 1.24 & 9.51 \\
\hline Asp, $t$ & 1.73 & 2.78 & 2.72 & 0.28 & 0.35 & 10.22 \\
\hline Gln, $g+$ & 1.69 & 3.67 & 2.26 & 0.28 & 0.62 & 9.55 \\
\hline Gln, $g-$ & 1.57 & 3.73 & 2.29 & 0.34 & 0.82 & 9.66 \\
\hline $\mathrm{Gln}, t$ & 1.59 & 3.80 & 2.44 & 0.28 & 0.72 & 9.62 \\
\hline Glu, $g+$ & 1.80 & 2.92 & 2.62 & 0.31 & 0.63 & 9.74 \\
\hline Glu, $g$ - & 1.85 & 2.81 & 2.63 & 0.20 & 0.91 & 9.66 \\
\hline Glu, $t$ & 1.81 & 3.04 & 2.66 & 0.25 & 0.71 & 9.71 \\
\hline Ile, $g+$ & 1.65 & 3.18 & 2.31 & 0.32 & 0.55 & 9.59 \\
\hline Ile, $g$ - & 1.58 & 3.31 & 2.41 & 0.32 & 0.76 & 9.64 \\
\hline Ile, $t$ & 1.66 & 3.45 & 2.31 & 0.33 & 0.47 & 9.61 \\
\hline Leu, $g+$ & 1.61 & 3.49 & 2.29 & 0.33 & 0.49 & 9.71 \\
\hline Leu, $g-$ & 1.57 & 3.38 & 2.25 & 0.30 & 0.93 & 9.40 \\
\hline Leu, $t$ & 1.60 & 3.69 & 2.45 & 0.33 & 0.63 & 9.82 \\
\hline Lys, $g^{+}$ & 1.54 & 3.87 & 2.12 & 0.28 & 0.57 & 9.83 \\
\hline Lys, $g$ - & 1.38 & 3.92 & 2.19 & 0.46 & 0.57 & 9.72 \\
\hline Lys, $t$ & 1.41 & 4.31 & 2.24 & 0.32 & 0.60 & 9.63 \\
\hline Phe, $g+$ & 1.61 & 3.68 & 2.25 & 0.38 & 0.73 & 9.59 \\
\hline Phe, $g$ - & 1.58 & 3.49 & 2.43 & 0.30 & 0.74 & 9.82 \\
\hline Phe, $t$ & 1.58 & 3.87 & 2.45 & 0.34 & 0.68 & 9.79 \\
\hline Ser, $g+$ & 1.47 & 3.58 & 2.41 & 0.29 & 0.58 & 9.61 \\
\hline Ser, $g-$ & 1.64 & 3.56 & 2.29 & 0.23 & 0.97 & 9.50 \\
\hline Ser, $t$ & 1.59 & 4.25 & 2.47 & 0.32 & 0.64 & 9.68 \\
\hline Thr, $g+$ & 1.48 & 3.32 & 2.35 & 0.30 & 0.62 & 9.62 \\
\hline Thr, g- & 1.61 & 3.42 & 2.35 & 0.25 & 0.89 & 9.58 \\
\hline Thr, $t$ & 1.62 & 3.84 & 2.40 & 0.39 & 0.27 & 9.86 \\
\hline $\operatorname{Trp}, g+$ & 1.64 & 3.85 & 2.29 & 0.41 & 0.80 & 9.59 \\
\hline Trp, $g-$ & 1.59 & 3.65 & 2.44 & 0.32 & 0.81 & 9.82 \\
\hline $\operatorname{Trp}, t$ & 1.58 & 3.93 & 2.48 & 0.35 & 0.69 & 9.87 \\
\hline Tyr, $g+$ & 1.63 & 3.69 & 2.27 & 0.38 & 0.75 & 9.58 \\
\hline Tyr, $g$ - & 1.59 & 3.51 & 2.44 & 0.31 & 0.76 & 9.82 \\
\hline Tyr, $t$ & 1.59 & 3.88 & 2.45 & 0.34 & 0.69 & 9.78 \\
\hline Val, $g+$ & 1.66 & 3.56 & 2.33 & 0.33 & 0.49 & 9.61 \\
\hline Val, $g-$ & 1.65 & 3.29 & 2.30 & 0.32 & 0.63 & 9.59 \\
\hline Val, $t$ & 1.59 & 3.41 & 2.39 & 0.32 & 0.77 & 9.65 \\
\hline
\end{tabular}


Effect of H-bonding on ${ }^{3} \mathrm{~J}$. Effects of H-bonding on the couplings also were studied using the Ace-Ala-NMe dipeptide analogue. Four water molecules were included in the calculation, each of which serves either as a H-bond donor to a carbonyl group or as an acceptor of an amide group. Typical $\mathrm{N}-\mathrm{H}---\mathrm{O}=\mathrm{C}$ H-bonding geometries in a helix and a sheet structure were used to construct these backbone-water H-bonds. To study the effect of the amide (or the preceding carbonyl) H-bond on the couplings, the distance between the amide $\mathrm{N}$ (or the carbonyl $\mathrm{O}$ ) atom and the $\mathrm{O}$ atom of the water $\mathrm{H}$-bonded to the amide group (or the carbonyl group) was varied from 2.8 to $3.2 \AA$ in a step of $0.1 \AA$, while the $\mathrm{N}-\mathrm{O}$ and $\mathrm{O}-\mathrm{O}$ distances in the other backbone-water H-bonds were kept fixed at $2.8 \AA$. In addition to the geometric constraints used in the above study of the effect of amino acid residue types, the water oxygen atom positions relative to the backbone are constrained in the partial geometry optimization. Calculation of the scalar coupling constants was then performed using the optimized geometry and the resulting couplings are presented in Table S2. The appreciable effect of the amide H-bond strength on ${ }^{3} J_{\mathrm{HN}, \mathrm{H} \alpha}$ is illustrated in Figure S1 for both helical and extended geometry.

Table S2. Calculated ${ }^{3} J$ couplings as a function of H-bond length in Ace-Ala-NMe.

\begin{tabular}{|c|c|c|c|c|c|c|}
\hline \multirow{2}{*}{ residue, $\mathrm{H}-$-bond distance } & \multicolumn{3}{|c|}{ couplings for $\alpha$-Helix $(\mathrm{Hz})$} & \multicolumn{3}{c|}{ couplings for $\beta$-Sheet $(\mathrm{Hz})$} \\
\cline { 2 - 7 } & ${ }^{3} J_{\mathrm{HN}-\mathrm{C}^{\prime}}$ & ${ }^{3} J_{\mathrm{HN}, \mathrm{C} \beta}$ & ${ }^{3} J_{\mathrm{HN}, \mathrm{H} \alpha}$ & ${ }^{3} J_{\mathrm{HN}-\mathrm{C}^{\prime}}$ & ${ }^{3} J_{\mathrm{HN}, \mathrm{C} \beta}$ & ${ }^{3} J_{\mathrm{HN}, \mathrm{H} \alpha}$ \\
\hline Ala, $\mathrm{N}_{\mathrm{i}}-\mathrm{O} 2.8 \AA$ & 0.93 & 3.74 & 3.40 & 0.18 & 1.44 & 8.72 \\
\hline Ala, $\mathrm{N}_{\mathrm{i}}-\mathrm{O} 2.9 \AA$ & 0.94 & 3.75 & 3.48 & 0.17 & 1.44 & 8.97 \\
\hline Ala, $\mathrm{N}_{\mathrm{i}}-\mathrm{O} 3.0 \AA$ & 0.95 & 3.75 & 3.54 & 0.16 & 1.43 & 9.16 \\
\hline Ala, $\mathrm{N}_{\mathrm{i}}-\mathrm{O} 3.1 \AA$ & 0.95 & 3.76 & 3.60 & 0.15 & 1.43 & 9.31 \\
\hline Ala, $\mathrm{N}_{\mathrm{i}}-\mathrm{O} 3.2 \AA$ & 0.96 & 3.75 & 3.63 & 0.14 & 1.42 & 9.43 \\
\hline Ala, $\mathrm{CO}_{\mathrm{i}-1}-\mathrm{O} 2.8 \AA$ & 0.93 & 3.74 & 3.40 & 0.18 & 1.44 & 8.72 \\
\hline Ala, $\mathrm{CO}_{\mathrm{i}-1}-\mathrm{O} 2.9 \AA$ & 0.93 & 3.76 & 3.39 & 0.17 & 1.44 & 8.72 \\
\hline Ala, $\mathrm{CO}_{\mathrm{i}-1}-\mathrm{O} 3.0 \AA$ & 0.93 & 3.77 & 3.38 & 0.17 & 1.44 & 8.72 \\
\hline Ala, $\mathrm{CO}_{\mathrm{i}-1}-\mathrm{O} 3.1 \AA$ & 0.93 & 3.78 & 3.38 & 0.16 & 1.44 & 8.71 \\
\hline Ala, $\mathrm{CO}_{\mathrm{i}-1}-\mathrm{O} 3.2 \AA$ & 0.93 & 3.79 & 3.37 & 0.15 & 1.44 & 8.71 \\
\hline
\end{tabular}




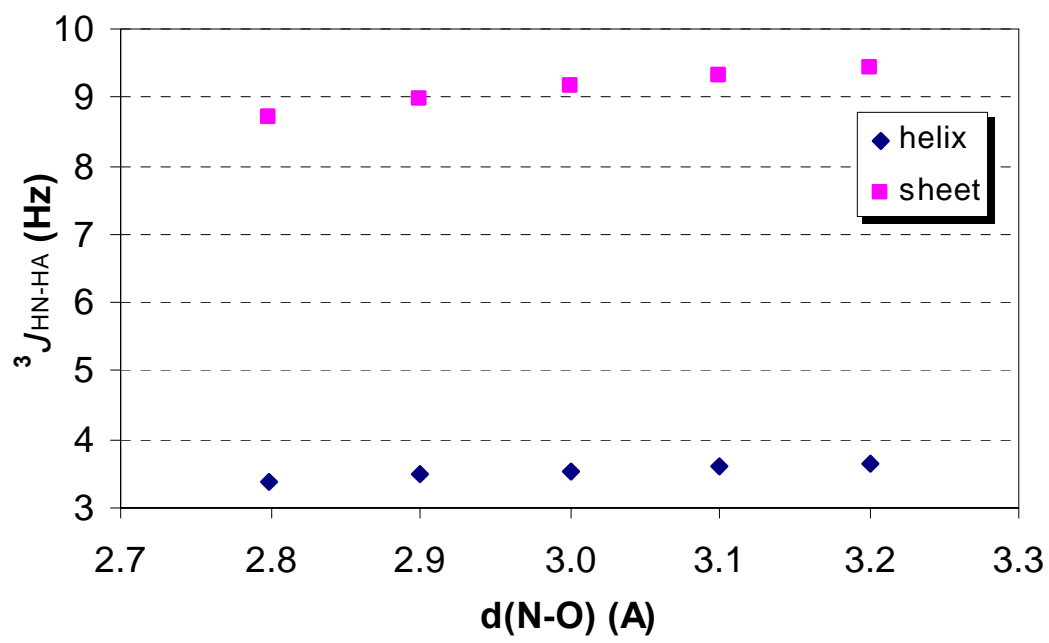

Figure S1. Effect of the amide H-bond length on ${ }^{3} J_{\mathrm{HN}, \mathrm{H} \alpha}$ computed for a helical and an extended (sheet) geometry of Ace-Ala-NMe.

Effect of bond angles on ${ }^{3} J$. To investigate how the bond angles of $\mathrm{C}_{\mathrm{i}-1}^{\prime}-\mathrm{N}_{\mathrm{i}}-\mathrm{C}^{\alpha}{ }_{\mathrm{i}}, \mathrm{N}_{\mathrm{i}}-\mathrm{C}^{\alpha} \mathrm{i}^{-}$ $\mathrm{C}^{\prime}{ }_{\mathrm{i}}$, and $\mathrm{N}_{\mathrm{i}}-\mathrm{C}^{\alpha}{ }_{\mathrm{i}}-\mathrm{C}^{\beta}{ }_{\mathrm{i}}$ affect the couplings, a series of calculations were performed using the Ace-Ala-NMe dipeptide analogue in both a helical and extended geometry. In these calculations, one of the above bond angles was independently varied (for $\mathrm{C}_{\mathrm{i}-1}^{\prime}-\mathrm{N}_{\mathrm{i}}-\mathrm{C}^{\alpha}{ }_{\mathrm{i}}$ from $115^{\circ}$ to $125^{\circ}$; for $\mathrm{N}_{\mathrm{i}}-\mathrm{C}^{\alpha}{ }_{\mathrm{i}}-\mathrm{C}^{\prime}{ }_{\mathrm{i}}$ and $\mathrm{N}_{\mathrm{i}}-\mathrm{C}^{\alpha}{ }_{\mathrm{i}}-\mathrm{C}^{\beta}{ }_{\mathrm{i}}$ from $104.5^{\circ}$ to $114.5^{\circ}$ ). The same geometry constraints as described in the above study of the effect of amino acid residue types were applied during the partial geometry optimization. The optimized geometry was then used for the coupling calculations and the computed couplings are presented in Table S3. Figure S2 shows the dependence of the couplings on the various angles for the helical geometry, whereas for extended structure the analogous graphs are shown in Figure S3. 
Table S3. Calculated ${ }^{3} J$ couplings as a function of the $\mathrm{C}^{\prime}{ }_{\mathrm{i}-1}-\mathrm{N}_{\mathrm{i}-}-\mathrm{C}^{\alpha}{ }_{\mathrm{i}}, \mathrm{N}_{\mathrm{i}}-\mathrm{C}^{\alpha}{ }_{\mathrm{i}-\mathrm{C}} \mathrm{C}_{\mathrm{i}}{ }$, and $\mathrm{N}_{\mathrm{i}-\mathrm{C}^{\alpha}}{ }_{\mathrm{i}-}$ $\mathrm{C}^{\beta}{ }_{\mathrm{i}}$ angles in Ace-Ala-NMe.

\begin{tabular}{|c|c|c|c|c|c|c|}
\hline \multirow{2}{*}{ 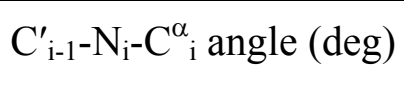 } & \multicolumn{3}{|c|}{ couplings for $\alpha$-Helix $(\mathrm{Hz})$} & \multicolumn{3}{|c|}{ couplings for $\beta$-Sheet $(\mathrm{Hz})$} \\
\hline & ${ }^{3} J_{\mathrm{HN}, \mathrm{C}^{\prime}}$ & ${ }^{3} J_{\mathrm{HN}, \mathrm{C} \beta}$ & ${ }^{3} J_{\mathrm{HN}, \mathrm{H} \alpha}$ & ${ }^{3} J_{\mathrm{HN}, \mathrm{C}^{\prime}}$ & ${ }^{3} J_{\mathrm{HN}, \mathrm{C} \beta}$ & ${ }^{3} J_{\mathrm{HN}, \mathrm{Ho}}$ \\
\hline 115 & 1.30 & 3.38 & 2.09 & 0.14 & 0.56 & 9.04 \\
\hline 116 & 1.33 & 3.50 & 2.14 & 0.18 & 0.60 & 9.16 \\
\hline 117 & 1.36 & 3.62 & 2.19 & 0.22 & 0.65 & 9.27 \\
\hline 118 & 1.40 & 3.73 & 2.24 & 0.26 & 0.70 & 9.39 \\
\hline 119 & 1.43 & 3.85 & 2.29 & 0.31 & 0.74 & 9.50 \\
\hline 120 & 1.46 & 3.97 & 2.34 & 0.35 & 0.79 & 9.61 \\
\hline 121 & 1.49 & 4.09 & 2.38 & 0.39 & 0.84 & 9.73 \\
\hline 122 & 1.51 & 4.20 & 2.43 & 0.44 & 0.89 & 9.84 \\
\hline 123 & 1.54 & 4.32 & 2.47 & 0.48 & 0.93 & 9.95 \\
\hline 124 & 1.57 & 4.43 & 2.51 & 0.53 & 0.98 & 10.06 \\
\hline 125 & 1.59 & 4.54 & 2.56 & 0.57 & 1.03 & 10.17 \\
\hline \multicolumn{7}{|l|}{$\mathrm{N}_{\mathrm{i}}-\mathrm{C}^{\alpha}{ }_{\mathrm{i}}-\mathrm{C}_{\mathrm{i}}^{\prime}$ angle $(\mathrm{deg})$} \\
\hline 104.5 & 1.32 & 3.82 & 2.19 & 0.49 & 0.80 & 9.06 \\
\hline 105.5 & 1.35 & 3.85 & 2.22 & 0.45 & 0.80 & 9.17 \\
\hline 106.5 & 1.38 & 3.88 & 2.25 & 0.43 & 0.80 & 9.28 \\
\hline 107.5 & 1.41 & 3.91 & 2.28 & 0.40 & 0.80 & 9.39 \\
\hline 108.5 & 1.43 & 3.94 & 2.31 & 0.37 & 0.79 & 9.50 \\
\hline 109.5 & 1.46 & 3.97 & 2.34 & 0.35 & 0.79 & 9.61 \\
\hline 110.5 & 1.48 & 4.00 & 2.36 & 0.33 & 0.79 & 9.72 \\
\hline 111.5 & 1.50 & 4.02 & 2.38 & 0.31 & 0.78 & 9.83 \\
\hline 112.5 & 1.51 & 4.05 & 2.41 & 0.28 & 0.78 & 9.94 \\
\hline 113.5 & 1.53 & 4.07 & 2.43 & 0.27 & 0.78 & 10.05 \\
\hline 114.5 & 1.54 & 4.10 & 2.44 & 0.25 & 0.77 & 10.15 \\
\hline \multicolumn{7}{|l|}{$\mathrm{N}_{\mathrm{i}}-\mathrm{C}^{\alpha}{ }_{\mathrm{i}}-\mathrm{C}^{\beta}{ }_{\mathrm{i}}$ angle $(\mathrm{deg})$} \\
\hline 104.5 & 1.28 & 4.19 & 2.04 & 0.35 & 1.00 & 9.01 \\
\hline 105.5 & 1.32 & 4.15 & 2.10 & 0.35 & 0.96 & 9.13 \\
\hline 106.5 & 1.35 & 4.11 & 2.16 & 0.35 & 0.91 & 9.25 \\
\hline 107.5 & 1.39 & 4.06 & 2.21 & 0.35 & 0.87 & 9.37 \\
\hline 108.5 & 1.42 & 4.02 & 2.28 & 0.35 & 0.83 & 9.49 \\
\hline 109.5 & 1.46 & 3.97 & 2.34 & 0.35 & 0.79 & 9.61 \\
\hline 110.5 & 1.49 & 3.92 & 2.40 & 0.35 & 0.75 & 9.73 \\
\hline 111.5 & 1.53 & 3.88 & 2.46 & 0.35 & 0.72 & 9.85 \\
\hline 112.5 & 1.57 & 3.83 & 2.53 & 0.34 & 0.69 & 9.97 \\
\hline 113.5 & 1.60 & 3.78 & 2.59 & 0.34 & 0.66 & 10.08 \\
\hline 114.5 & 1.64 & 3.74 & 2.66 & 0.33 & 0.63 & 10.20 \\
\hline
\end{tabular}




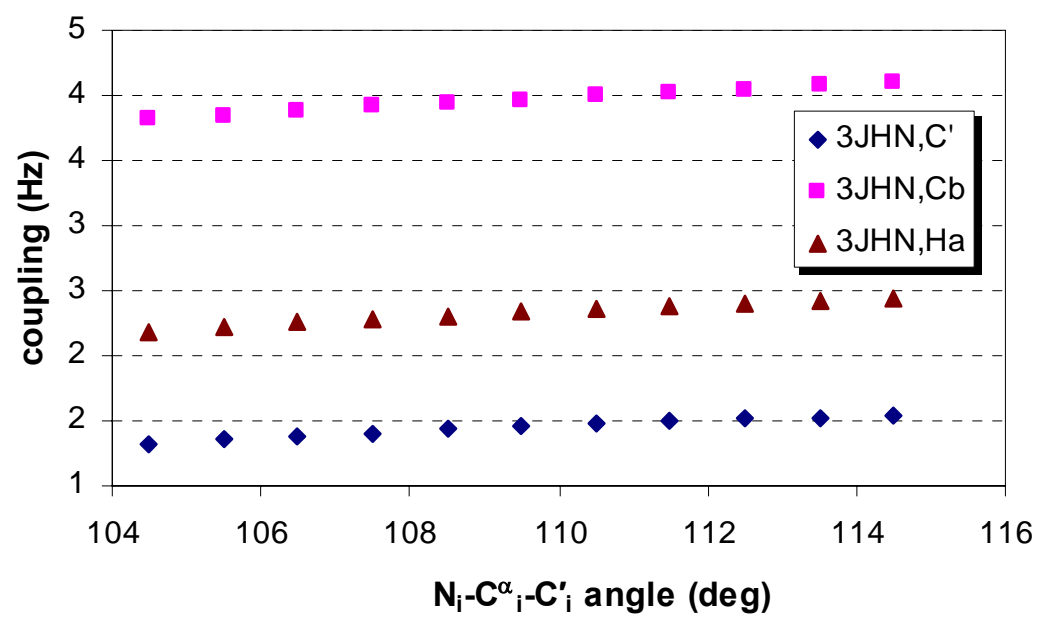

Figure S2. Dependence of various ${ }^{3} J$ couplings on the $\mathrm{N}_{\mathrm{i}}-\mathrm{C}^{\alpha}{ }_{\mathrm{i}}-\mathrm{C}_{\mathrm{i}}^{\prime}$ bond angle in a helical geometry of Ace-Ala-NMe.
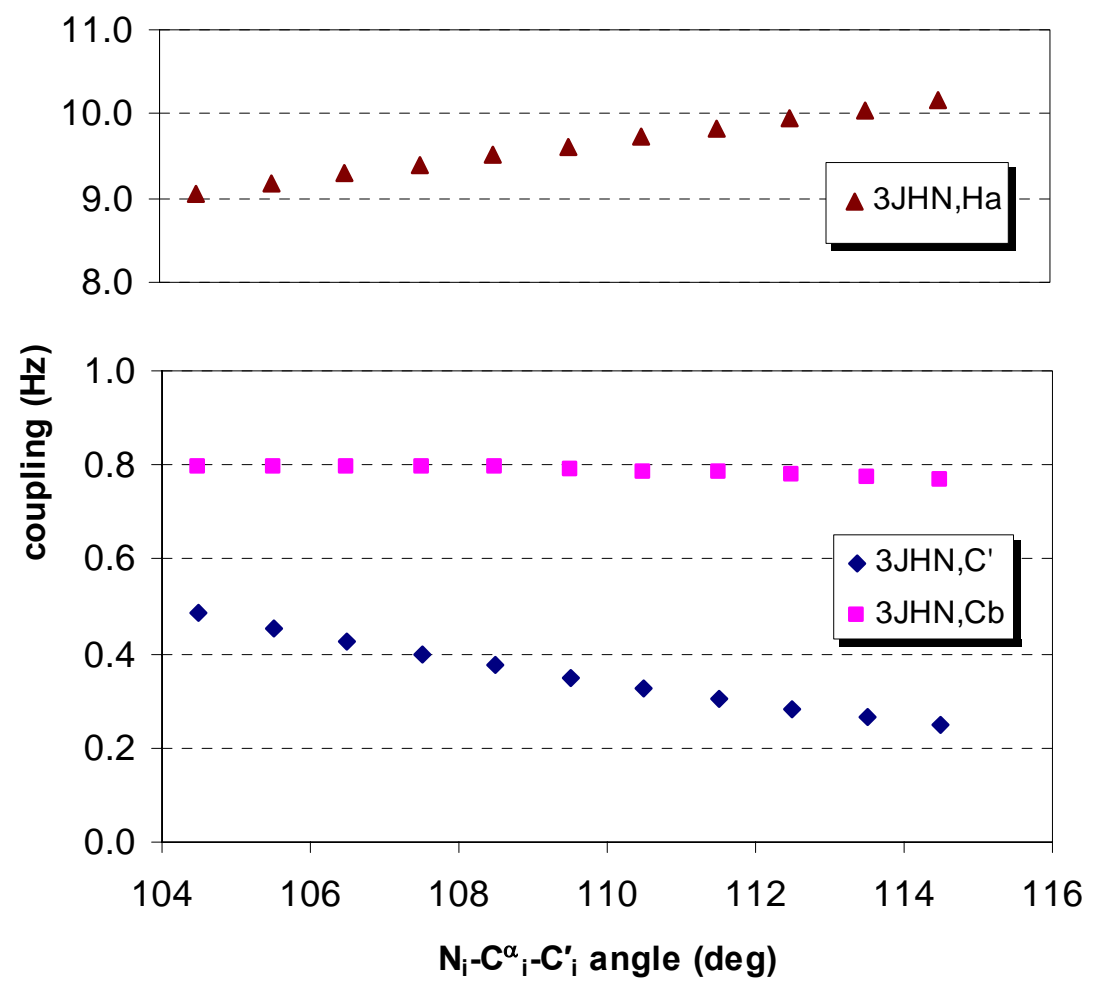

Figure S3. Dependence of various ${ }^{3} J$ couplings on the $\mathrm{N}_{\mathrm{i}}-\mathrm{C}^{\alpha}{ }_{\mathrm{i}}-\mathrm{C}_{\mathrm{i}}^{\prime}$ bond angle in an extended geometry of Ace-Ala-NMe. 
Table S4. $J$ couplings $[\mathrm{Hz}]$ and relaxation rates $\left[\mathrm{s}^{-1}\right]$ from individual experiments.

\begin{tabular}{|c|c|c|c|c|c|c|c|c|c|c|c|c|c|}
\hline & \multicolumn{4}{|c|}{$\begin{array}{c}\text { ct-MQ }\left({ }^{1} H^{N} \cdot{ }^{13} \mathrm{Ca}\right)+S Q\left({ }^{1} H^{N}\right)- \\
H N C A\end{array}$} & \multicolumn{3}{|c|}{ CT-HMQC-J } & \multicolumn{2}{|c|}{$\begin{array}{c}\mathrm{MQ}\left({ }^{1} \mathrm{H}^{\mathrm{N}} \cdot{ }^{13} \mathrm{C}^{\alpha}\right)- \\
\mathrm{HNCA}\end{array}$} & \multirow{3}{*}{$\begin{array}{l}\text { E.COSY } \\
{ }^{3} J_{\mathrm{HN}, \mathrm{C} \beta} \\
\end{array}$} & \multicolumn{2}{|c|}{\begin{tabular}{|c|} 
ct-MQ $\left({ }^{1} H^{N}\right.$. \\
$\left.{ }^{13} \mathrm{C}^{\alpha}\right)-\mathrm{HNCA}$
\end{tabular}} & \multirow{2}{*}{ E.COSY } \\
\hline & \multicolumn{2}{|c|}{$\kappa=1$} & \multicolumn{2}{|c|}{$\kappa=2$} & & & & & & & & & \\
\hline & ${ }^{3} J_{\mathrm{HN}, \mathrm{H \alpha}}$ & ${ }^{1} J_{\mathrm{C} \alpha, \mathrm{H} \alpha}$ & ${ }^{3} J_{\mathrm{HN}, \mathrm{H} \alpha}$ & ${ }^{1} J_{\mathrm{C} \alpha, \mathrm{H} \alpha}$ & ${ }^{3} J_{\mathrm{HN}, \mathrm{H} \alpha}$ & $R_{\mathrm{SQ}}$ & $R_{\mathrm{MQ}}$ & ${ }^{3} J_{\mathrm{HN}, \mathrm{C} \beta}$ & ${ }^{1} J_{\mathrm{C} \alpha, \mathrm{C \beta}}$ & & ${ }^{3} J_{\mathrm{HN}, \mathrm{C}^{\prime}}$ & ${ }^{1} J_{\mathrm{C} \alpha, \mathrm{C}^{\prime}}$ & ${ }^{3} J_{\mathrm{HN}, \mathrm{C}^{\prime}}$ \\
\hline \multicolumn{14}{|l|}{ Res\# } \\
\hline 3 & 9.58 & \begin{tabular}{|l|}
145.5 \\
\end{tabular} & 9.49 & 144.6 & 10.01 & 4.0 & 20.3 & 0.77 & 32.6 & 0.66 & 0.89 & 51.8 & 0.62 \\
\hline 4 & 9.88 & 139.8 & 9.53 & 139.6 & 9.85 & 4.3 & 20 & 1.1 & 33.9 & 1.11 & 0.16 & 53.1 & 0.28 \\
\hline 5 & 9.52 & 142.2 & 9.56 & 142.2 & 9.62 & 4.7 & 21.8 & 0.57 & 34.7 & 0.55 & 0.7 & 52.0 & 0.71 \\
\hline 6 & 10.01 & 140.0 & 9.6 & 139.7 & 9.75 & 3.4 & 22.3 & 1.17 & 35.3 & 0.97 & 0.16 & 53.3 & 0.29 \\
\hline 7 & 9.68 & \begin{tabular}{|l|}
140.4 \\
\end{tabular} & 9.52 & 140.2 & 9.54 & 4.6 & 19.8 & 1.37 & 36.2 & 1.14 & 0.25 & 53.4 & 0.22 \\
\hline 8 & 9.71 & 140.3 & 9.66 & 139.8 & 10.08 & 3.5 & 17.7 & 0.75 & 42.5 & 0.93 & 0.75 & 53.7 & 0.56 \\
\hline 9 & & & & & & & & & & & & 50.9 & \\
\hline 10 & 4.88 & \begin{tabular}{|l|}
147.2 \\
\end{tabular} & 4.88 & 147.1 & 5.11 & 2.0 & 22.3 & 2.87 & 33.5 & 2.70 & 0.91 & 52.8 & 0.97 \\
\hline 11 & 10.61 & 141.6 & 10.24 & 141.0 & 10.2 & 2.7 & 18.2 & 0.91 & 37.9 & 0.82 & 0.32 & 53.5 & 0.39 \\
\hline 12 & 7.76 & \begin{tabular}{|l|}
141.6 \\
\end{tabular} & 7.83 & \begin{tabular}{|l|}
141.3 \\
\end{tabular} & 7.89 & 2.0 & 18.1 & 1.5 & 34.8 & 1.27 & 0.78 & 53.3 & 0.86 \\
\hline 13 & & & & & 9.94 & 8.5 & 11.5 & 0.54 & 35 & 0.37 & 0.88 & 52.5 & 1.25 \\
\hline 14 & & & & & & & & & & & & 51.5 & \\
\hline 15 & 8.44 & 144.4 & & & & & & 0.35 & 33.5 & & 1.64 & 52.5 & 1.69 \\
\hline 16 & 6.8 & \begin{tabular}{|l|}
141.2 \\
\end{tabular} & 6.72 & 141.0 & 6.75 & 2.0 & 17.6 & 0.25 & 38.6 & 0.12 & 2.54 & 51.3 & 2.69 \\
\hline 17 & & & & & 8.98 & 2.0 & 17.3 & 0.18 & 36.5 & 0.14 & 1.49 & 52.6 & 1.56 \\
\hline 18 & 6.8 & 142.8 & 6.61 & 142.6 & 6.77 & 2.0 & 18.7 & 0.18 & 37.9 & 23 & 2.49 & 51.4 & 2.89 \\
\hline 19 & 9.4 & \begin{tabular}{|l|}
143.2 \\
\end{tabular} & & & 9.37 & 2.3 & 20.5 & 1.32 & 35.3 & 0.99 & 0.61 & 52.4 & 0.45 \\
\hline 20 & 7.22 & 143.1 & 7.21 & 142.9 & 7.32 & 2.0 & 17.6 & 0.11 & 33.7 & 0.09 & 2.17 & 52.0 & 2.24 \\
\hline 21 & 4.11 & 145.7 & 4.39 & 145.9 & 4.39 & 2.0 & 22.2 & 2.67 & 33.2 & 2.60 & 1.64 & 53.4 & 1.98 \\
\hline 22 & 6.3 & \begin{tabular}{|l|}
140.6 \\
\end{tabular} & 6.38 & 140.8 & 6.33 & 2.0 & 15.9 & 0.24 & 35.8 & 0.19 & 2.66 & 52.9 & 2.92 \\
\hline 23 & 3.0 & 146.9 & 3.09 & 146.6 & 3.09 & 2.2 & 21.6 & 3.41 & 33.5 & 3.08 & 2.16 & 53.4 & 2.03 \\
\hline 24 & 3.87 & 148.7 & 3.85 & 148.8 & 4.07 & 2.1 & 16.4 & 3.19 & 33.4 & 3.01 & 1.52 & 52.3 & 1.54 \\
\hline 25 & 5.35 & 147 & & & & & & 3.35 & 35.9 & & 0.77 & 50.7 & 0.72 \\
\hline 26 & 4.3 & 148.4 & 4.3 & 148.2 & 4.5 & 2.0 & 17.8 & 3.27 & 33.5 & 3.23 & 1.00 & 53.0 & 1.38 \\
\hline 27 & 1.84 & \begin{tabular}{|l|}
148.3 \\
\end{tabular} & 2.03 & 148.4 & & & & 3.12 & 33.4 & 2.85 & 2.52 & 53.0 & 2.98 \\
\hline 28 & 3.3 & 148 & 3.28 & \begin{tabular}{|l|}
148.3 \\
\end{tabular} & 4.08 & 2.0 & 19 & 2.64 & 33.1 & 3.00 & 1.84 & 52.4 & \\
\hline 29 & 4.66 & 150 & 4.71 & 149.8 & 4.99 & 2.0 & 15.7 & 3.21 & 33.2 & 3.16 & 0.67 & 52.1 & 0.94 \\
\hline 30 & 4.44 & 144.3 & 4.57 & 144.7 & 5.1 & 5.2 & 18.1 & 2.88 & 33.2 & 2.88 & 0.56 & 54.7 & 0.77 \\
\hline 31 & 4.06 & \begin{tabular}{|l|}
146.9 \\
\end{tabular} & 3.99 & \begin{tabular}{|l|}
147.1 \\
\end{tabular} & 4.24 & 2 & \begin{tabular}{|l|}
19.7 \\
\end{tabular} & 2.93 & 33.4 & 2.65 & 1.13 & 53.1 & 1.20 \\
\hline 32 & & & & & 5.25 & 3.8 & 16.7 & 3.2 & 33.7 & 2.99 & 0.92 & 52.6 & 0.98 \\
\hline 33 & 2.98 & 152.1 & 3.1 & 1526 & 3.37 & 2.1 & 19 & 3.44 & 32.0 & 3.26 & 1.41 & 53.2 & 1.74 \\
\hline 34 & 3.46 & \begin{tabular}{|l|}
146.6 \\
\end{tabular} & 3.67 & 146.5 & 3.75 & 2.4 & 18.3 & 3.47 & 33.3 & 3.31 & 1.19 & 53.6 & 1.35 \\
\hline 35 & 3.7 & 150.8 & & & & & & 3.2 & 34.6 & 3.00 & 1.3 & 52.5 & 1.31 \\
\hline 36 & 3.36 & \begin{tabular}{|l|}
149.7 \\
\end{tabular} & 3.32 & 149.8 & 3.98 & 2.3 & 16.2 & 2.41 & 36.2 & 2.21 & 1.9 & 52.6 & 2.31 \\
\hline 37 & 9.03 & \begin{tabular}{|l|}
140.3 \\
\end{tabular} & 9.00 & 138.8 & 9.56 & 2.0 & 15.3 & 1.71 & 36.5 & 1.39 & \begin{tabular}{|l|}
-0.07 \\
\end{tabular} & 53.7 & 0.11 \\
\hline 38 & & & & & & & & & & & & 52.8 & \\
\hline 39 & 8.97 & \begin{tabular}{|l|}
142.2 \\
\end{tabular} & 9.03 & 141.9 & 8.96 & 2.0 & 15.7 & 1.57 & 34.8 & 1.41 & 0.18 & 53.6 & 0.19 \\
\hline 40 & 8.3 & \begin{tabular}{|l|}
142.2 \\
\end{tabular} & 8.29 & 141.9 & 8.5 & 2.3 & 14.4 & 0.42 & 37.8 & 0.29 & 1.81 & 53.1 & 1.78 \\
\hline 41 & & & & & & & & & & & & 51.5 & \\
\hline 42 & 8.71 & \begin{tabular}{|l|}
142.1 \\
\end{tabular} & 8.7 & 141.7 & 8.76 & 2.0 & 18.8 & 1.68 & 34.4 & 1.61 & \begin{tabular}{|l|}
0.04 \\
\end{tabular} & 52.6 & 0.10 \\
\hline 43 & 8.46 & \begin{tabular}{|l|}
145.8 \\
\end{tabular} & & & 8.84 & 2.5 & 20.2 & & 31.9 & 1.64 & -0.15 & 52.5 & 0.06 \\
\hline
\end{tabular}


- S8 -

\begin{tabular}{|c|c|c|c|c|c|c|c|c|c|c|c|c|c|}
\hline 44 & 8.94 & 139.2 & & & 8.93 & 2.0 & 19 & 0.16 & 37.4 & 0.12 & 1.81 & 52.5 & 1.50 \\
\hline 45 & 8.74 & 142.1 & 8.74 & 141.6 & 8.85 & 2.0 & 19.9 & 0.34 & 33.1 & 0.19 & 1.21 & 54.2 & 1.14 \\
\hline 46 & 9.75 & 143.1 & 9.69 & 142.4 & 9.96 & 2.4 & 18.8 & 0.79 & 37.4 & 0.75 & 0.64 & 53.9 & 0.73 \\
\hline 47 & 3.94 & 148.1 & 3.97 & 147.9 & 4.15 & 2.0 & 20 & 2.86 & 33.5 & 2.76 & 1.496 & 52.8 & 1.38 \\
\hline 48 & & & 4.21 & 149.5 & 4.43 & 2.0 & 14.2 & 2.88 & 33.3 & 2.58 & 1.46 & 52.9 & 1.49 \\
\hline 49 & 10.01 & 138.5 & 10.13 & 138.6 & 10.02 & 2.5 & 17.7 & 1.42 & 39.3 & 1.39 & -0.01 & 53.1 & 0.08 \\
\hline 50 & & & & & 7.05 & 2.0 & 20.0 & 1.03 & 39.4 & 0.81 & 2.90 & 52.0 & 3.14 \\
\hline 51 & 10.16 & 142.8 & 10.1 & 141.7 & 10.36 & 5.7 & 16.4 & 0.89 & 36.7 & 0.64 & 0.31 & 52.8 & 0.38 \\
\hline 52 & 9.31 & 145.9 & & & 9.58 & 2.3 & 23.8 & 1.69 & 33.3 & 1.32 & 0.01 & 51.5 & 0.27 \\
\hline 53 & 9.77 & 143.9 & 9.73 & 143.3 & 9.63 & 2.7 & 20.4 & 0.30 & 37.1 & 0.29 & 0.97 & 51.6 & 1.22 \\
\hline 54 & 10.23 & 140.8 & 9.92 & 140.2 & 9.84 & 4.3 & 20.4 & 0.21 & 34.9 & 0.21 & 1.11 & 52.7 & 1.04 \\
\hline 55 & 9.87 & 142.6 & 9.75 & 142.1 & 10.00 & 3.5 & 22.1 & 0.98 & 38.3 & 0.92 & 0.23 & 52.3 & 0.29 \\
\hline 56 & 8.22 & 143.7 & 8.19 & 1432 & 8.40 & 2.0 & 18.7 & 2.29 & 34.5 & 2.08 & 0.12 & 54.0 & 0.34 \\
\hline & & & & & & & & & & & & & \\
\hline Ubiquitin & & & & & & & & & & & & & \\
\hline 46 & & & & & 6.47 & & & & & 0.27 & & & 3.58 \\
\hline 60 & & & & & 7.26 & & & & & 0.80 & & & 2.52 \\
\hline 64 & & & & & 7.19 & & & & & 1.35 & & & 1.63 \\
\hline
\end{tabular}


Table S5. Backbone ${ }^{3} J$ couplings in GB3, averaged over multiple measurements and used for all analyses. ${ }^{\text {a }}$

\begin{tabular}{|c|c|c|c|}
\hline Residue & ${ }^{3} J_{\mathrm{HN}, \mathrm{H} \alpha}$ & ${ }^{3} J_{\mathrm{HN}, \mathrm{C} \beta}$ & ${ }^{3} J_{\mathrm{HN}, \mathrm{C}^{\prime}}$ \\
\hline Y3 & 9.8 & 0.74 & 0.75 \\
\hline K4 & 9.8 & 1.16 & 0.22 \\
\hline L5 & 9.6 & 0.57 & 0.71 \\
\hline V6 & 9.8 & 1.10 & 0.22 \\
\hline I7 & 9.6 & 1.29 & 0.23 \\
\hline N8 & 9.9 & 0.87 & 0.66 \\
\hline K10 & 5.0 & 2.87 & 0.94 \\
\hline T11 & 10.3 & 0.89 & 0.35 \\
\hline L12 & 7.8 & 1.42 & 0.82 \\
\hline K13 & 9.9 & 0.46 & 1.06 \\
\hline E15 & 8.4 & 0.35 & 1.67 \\
\hline T16 & 6.8 & 0.19 & 2.61 \\
\hline T17 & 9.0 & 0.17 & 1.52 \\
\hline T18 & 6.7 & 0.21 & 2.69 \\
\hline K19 & 9.4 & 1.19 & 0.53 \\
\hline A20 & 7.3 & 0.10 & 2.20 \\
\hline V21 & 4.3 & 2.72 & 1.81 \\
\hline D22 & 6.3 & 0.22 & 2.79 \\
\hline A23 & 3.1 & 3.34 & 2.10 \\
\hline E24 & 4.0 & 3.19 & 1.53 \\
\hline T25 & 5.4 & 3.35 & 0.74 \\
\hline A26 & 4.4 & 3.35 & 1.19 \\
\hline K28 & 3.7 & 2.91 & 1.84 \\
\hline A29 & 4.8 & 3.28 & 0.81 \\
\hline F30 & 4.8 & 2.96 & 0.67 \\
\hline K31 & 4.1 & 2.87 & 1.17 \\
\hline Q32 & 5.3 & 3.18 & 0.95 \\
\hline Y33 & 3.2 & 3.45 & 1.58 \\
\hline A34 & 3.7 & 3.49 & 1.27 \\
\hline N35 & 3.7 & 3.20 & 1.31 \\
\hline D36 & 3.7 & 2.38 & 2.11 \\
\hline N37 & 9.3 & 1.59 & 0.02 \\
\hline V39 & 9.0 & 1.53 & 0.19 \\
\hline D40 & 8.4 & 0.36 & 1.80 \\
\hline V42 & 8.7 & 1.69 & 0.07 \\
\hline & & & \\
\hline
\end{tabular}




\begin{tabular}{|c|c|c|c|}
\hline W43 & 8.7 & 1.74 & -0.05 \\
\hline T44 & 8.9 & 0.14 & 1.66 \\
\hline Y45 & 8.8 & 0.27 & 1.17 \\
\hline D46 & 9.8 & 0.79 & 0.69 \\
\hline D47 & 4.1 & 2.89 & 1.44 \\
\hline A48 & 4.3 & 2.81 & 1.48 \\
\hline T49 & 10.0 & 1.45 & 0.03 \\
\hline K50 & 7.0 & 0.95 & 3.02 \\
\hline T51 & 10.3 & 0.78 & 0.35 \\
\hline F52 & 9.4 & 1.55 & 0.14 \\
\hline T53 & 9.7 & 0.30 & 1.10 \\
\hline V54 & 10.0 & 0.22 & 1.08 \\
\hline T55 & 9.9 & 0.98 & 0.26 \\
\hline E56 & 8.3 & 2.25 & 0.23 \\
\hline 3
\end{tabular}

a ${ }^{3} J_{\mathrm{HN}, \mathrm{H \alpha}}$ values represent the average over couplings derived from the CT$\mathrm{MQ}\left({ }^{1} \mathrm{H}^{\mathrm{N}},{ }^{13} \mathrm{C}^{\alpha}\right)+\mathrm{SQ}\left({ }^{1} \mathrm{H}^{\mathrm{N}}\right)$-HNCA spectra (averaged over the results from spectra recorded with $\kappa=1$ and $\kappa=2$ ), and $J$-modulated HMQC spectra. Based on the pairwise rmsd between these two sets of values, the error in their averaged value equals $0.14 \mathrm{~Hz} .{ }^{3} J_{\mathrm{HN}, \mathrm{C} \beta}$ values represent the average over couplings derived from the CT-MQ $\left({ }^{1} \mathrm{H}^{\mathrm{N}},{ }^{13} \mathrm{C}^{\alpha}\right)-\mathrm{HNCA}$ spectrum, and a HNCA[CB] E.COSY measurement, where the ${ }^{3} J$ values were scaled by 1.06 prior to averaging with the MQ-derived values. Based on the pairwise rmsd between these two sets of values, the random error in their averaged value equals $0.07 \mathrm{~Hz} .{ }^{3} J_{\mathrm{HN}, \mathrm{C}^{\prime}}$ values represent the average over couplings derived from a CT-MQ $\left({ }^{1} \mathrm{H}^{\mathrm{N}},{ }^{13} \mathrm{C}^{\alpha}\right)-\mathrm{HNCA}$ spectrum, and a $\mathrm{HNCA}\left[\mathrm{C}^{\prime}\right]$ E.COSY measurement. Based on the pairwise rmsd between these two sets of values, the random error in their averaged value equals $0.1 \mathrm{~Hz}$. 
Table S6. Karplus coefficients and statistical values from fits.

\begin{tabular}{|c|c|c|c|c|c|c|c|c|}
\hline $\begin{array}{l}\text { fitting } \\
\text { model }\end{array}$ & \# res & $\begin{array}{c}\mathrm{pdb} \\
\text { code }^{\mathrm{a}}\end{array}$ & ${ }^{3} J$ type & $\begin{array}{c}\mathrm{rmsd} \\
{[\mathrm{Hz}]}\end{array}$ & $R^{b}$ & $A[\mathrm{~Hz}]$ & $B[\mathrm{~Hz}]$ & $\mathrm{C}[\mathrm{Hz}]^{\mathrm{c}}$ \\
\hline \multirow[t]{3}{*}{ rigid } & $47+3^{d}$ & 1P7E & $\mathrm{H}^{\mathrm{N}}, \mathrm{H}^{\alpha}$ & 0.44 & 0.983 & 7.91 & -1.28 & 0.71 \\
\hline & & & $\mathrm{H}^{\mathrm{N}}, \mathrm{C}^{\prime}$ & 0.34 & 0.921 & 4.05 & -1.09 & 0.10 \\
\hline & & & $\mathrm{H}^{\mathrm{N}}, \mathrm{C}^{\beta}$ & 0.26 & 0.974 & 3.46 & -0.52 & 0.16 \\
\hline \multirow[t]{3}{*}{ rigid } & $47+3$ & 1P7F & $\mathrm{H}^{\mathrm{N}}, \mathrm{H}^{\alpha}$ & 0.45 & 0.983 & 8.00 & -1.27 & 0.62 \\
\hline & & & $\mathrm{H}^{\mathrm{N}}, \mathrm{C}^{\prime}$ & 0.32 & 0.929 & 4.09 & -1.10 & 0.11 \\
\hline & & & $\mathrm{H}^{\mathrm{N}}, \mathrm{C}^{\beta}$ & 0.28 & 0.970 & 3.41 & -0.48 & 0.17 \\
\hline \multirow[t]{3}{*}{ rigid } & $47+3$ & $1 I G D$ & $\mathrm{H}^{\mathrm{N}}, \mathrm{H}^{\alpha}$ & 0.68 & 0.961 & 7.95 & -1.23 & 0.64 \\
\hline & & & $\mathrm{H}^{\mathrm{N}}, \mathrm{C}^{\prime}$ & 0.43 & 0.869 & 3.87 & -1.15 & 0.19 \\
\hline & & & $\mathrm{H}^{\mathrm{N}}, \mathrm{C}^{\beta}$ & 0.33 & 0.958 & 3.26 & -0.38 & 0.25 \\
\hline \multirow[t]{3}{*}{ rigid } & $47+3$ & 2OED & $\mathrm{H}^{\mathrm{N}}, \mathrm{H}^{\alpha}$ & 0.42 & 0.985 & 7.97 & -1.26 & 0.63 \\
\hline & & & $\mathrm{H}^{\mathrm{N}}, \mathrm{C}^{\prime}$ & 0.31 & 0.933 & 4.12 & -1.10 & 0.11 \\
\hline & & & $\mathrm{H}^{\mathrm{N}}, \mathrm{C}^{\beta}$ & 0.25 & 0.976 & 3.51 & -0.53 & 0.14 \\
\hline \multirow[t]{3}{*}{$\sigma=10^{\circ}$} & $35^{\mathrm{e}}+3$ & 1IGD & $\mathrm{H}^{\mathrm{N}}, \mathrm{H}^{\alpha}$ & 0.58 & 0.968 & 8.28 & -1.24 & 0.53 \\
\hline & & & $\mathrm{H}^{\mathrm{N}}, \mathrm{C}^{\prime}$ & 0.39 & 0.913 & 4.38 & -1.09 & -0.04 \\
\hline & & & $\mathrm{H}^{\mathrm{N}}, \mathrm{C}^{\beta}$ & 0.32 & 0.955 & 3.53 & -0.44 & 0.14 \\
\hline \multirow[t]{3}{*}{$\sigma=0^{\circ}$} & $35^{\mathrm{e}}+3$ & 2OED & $\mathrm{H}^{\mathrm{N}}, \mathrm{H}^{\alpha}$ & 0.31 & 0.991 & 8.17 & -1.25 & 0.42 \\
\hline & & & $\mathrm{H}^{\mathrm{N}}, \mathrm{C}^{\prime}$ & 0.28 & 0.956 & 4.26 & -1.06 & 0.08 \\
\hline & & & $\mathrm{H}^{\mathrm{N}}, \mathrm{C}^{\beta}$ & 0.20 & 0.983 & 3.62 & -0.58 & 0.13 \\
\hline \multirow[t]{3}{*}{$\sigma=10^{\circ}$} & $35^{\mathrm{e}}+3$ & 2OED & $\mathrm{H}^{\mathrm{N}}, \mathrm{H}^{\alpha}$ & 0.31 & 0.991 & 8.69 & -1.27 & 0.16 \\
\hline & & & $\mathrm{H}^{\mathrm{N}}, \mathrm{C}^{\prime}$ & 0.28 & 0.956 & 4.53 & -1.08 & -0.05 \\
\hline & & & $\mathrm{H}^{\mathrm{N}}, \mathrm{C}^{\beta}$ & 0.20 & 0.983 & 3.85 & -0.59 & 0.02 \\
\hline \multirow[t]{3}{*}{$\sigma=20^{\circ}$} & $35^{\mathrm{e}}+3$ & 2OED & $\mathrm{H}^{\mathrm{N}}, \mathrm{H}^{\alpha}$ & 0.31 & 0.991 & 10.43 & -1.32 & -0.71 \\
\hline & & & $\mathrm{H}^{\mathrm{N}}, \mathrm{C}^{\prime}$ & 0.28 & 0.956 & 5.44 & -1.13 & -0.51 \\
\hline & & & $\mathrm{H}^{\mathrm{N}}, \mathrm{C}^{\beta}$ & 0.20 & 0.983 & 4.63 & -0.62 & -0.37 \\
\hline \multirow[t]{3}{*}{ motion $^{f}$} & $47+3$ & 2OED & $\mathrm{H}^{\mathrm{N}}, \mathrm{H}^{\alpha}$ & 0.49 & 0.980 & 8.82 & -1.33 & 0.20 \\
\hline & & & $\mathrm{H}^{\mathrm{N}}, \mathrm{C}^{\prime}$ & 0.35 & 0.918 & 4.47 & -1.09 & -0.09 \\
\hline & & & $\mathrm{H}^{\mathrm{N}}, \mathrm{C}^{\beta}$ & 0.28 & 0.972 & 3.88 & -0.50 & -0.07 \\
\hline \multirow[t]{3}{*}{ motion $^{g}$} & $49^{h}+3$ & 1IGD & $\mathrm{H}^{\mathrm{N}}, \mathrm{H}^{\alpha}$ & 0.63 & 0.973 & $8.28^{g}$ & -1.29 & 0.74 \\
\hline & & & $\mathrm{H}^{\mathrm{N}}, \mathrm{C}^{\prime}$ & 0.42 & 0.886 & $4.38^{g}$ & -1.05 & -0.13 \\
\hline & & & $\mathrm{H}^{\mathrm{N}}, \mathrm{C}^{\beta}$ & 0.38 & 0.95 & $3.53^{g}$ & 0.04 & -0.13 \\
\hline \multirow[t]{3}{*}{ motion $^{i}$} & $47+3$ & 2OED & $\mathrm{H}^{\mathrm{N}}, \mathrm{H}^{\alpha}$ & 0.41 & 0.987 & $8.17^{i}$ & -1.33 & 0.51 \\
\hline & & & $\mathrm{H}^{\mathrm{N}}, \mathrm{C}^{\prime}$ & 0.32 & 0.936 & $4.26^{i}$ & -1.11 & 0.02 \\
\hline & & & $\mathrm{H}^{\mathrm{N}}, \mathrm{C}^{\beta}$ & 0.24 & 0.980 & $3.62^{i}$ & -0.49 & 0.08 \\
\hline \multirow[t]{3}{*}{ motion $^{i}$} & $49^{h}+3$ & 2OED & $\mathrm{H}^{\mathrm{N}}, \mathrm{H}^{\alpha}$ & 0.44 & 0.985 & $8.17^{i}$ & -1.18 & 0.63 \\
\hline & & & $\mathrm{H}^{\mathrm{N}}, \mathrm{C}^{\prime}$ & 0.34 & 0.926 & $4.26^{i}$ & -1.01 & 0.01 \\
\hline & & & $\mathrm{H}^{\mathrm{N}}, \mathrm{C}^{\beta}$ & 0.26 & 0.976 & $3.62^{i}$ & -0.41 & 0.02 \\
\hline
\end{tabular}




\begin{tabular}{|c|c|c|c|c|c|c|c|c|}
\hline motion $^{g}$ & $47+3$ & 2OED & $\mathrm{H}^{\mathrm{N}}, \mathrm{H}^{\alpha}$ & 0.38 & 0.989 & $8.69^{9}$ & -1.14 & 0.39 \\
\hline & & & $\mathrm{H}^{\mathrm{N}}, \mathrm{C}^{\prime}$ & 0.31 & 0.938 & $4.53^{g}$ & -1.09 & -0.09 \\
\hline & & & $\mathrm{H}^{\mathrm{N}}, \mathrm{C}^{\beta}$ & 0.23 & 0.981 & $3.85^{\mathrm{g}}$ & -0.47 & -0.08 \\
\hline \multirow[t]{3}{*}{ motion $^{g}$} & $49^{\mathrm{h}}+3$ & 2OED & $\mathrm{H}^{\mathrm{N}}, \mathrm{H}^{\alpha}$ & 0.38 & 0.988 & $8.69^{9}$ & -1.01 & 0.47 \\
\hline & & & $\mathrm{H}^{\mathrm{N}}, \mathrm{C}^{\prime}$ & 0.33 & 0.931 & $4.53^{g}$ & -1.06 & -0.08 \\
\hline & & & $\mathrm{H}^{\mathrm{N}}, \mathrm{C}^{\beta}$ & 0.23 & 0.982 & $3.85^{\mathrm{g}}$ & -0.40 & -0.12 \\
\hline \multirow[t]{3}{*}{ motion $^{\mathrm{j}}$} & $47+3$ & 2OED & $\mathrm{H}^{\mathrm{N}}, \mathrm{H}^{\alpha}$ & 0.42 & 0.986 & $10.43^{j}$ & -1.27 & -0.65 \\
\hline & & & $\mathrm{H}^{\mathrm{N}}, \mathrm{C}^{\prime}$ & 0.36 & 0.921 & $5.44^{j}$ & -1.05 & -0.53 \\
\hline & & & $\mathrm{H}^{\mathrm{N}}, \mathrm{C}^{\beta}$ & 0.26 & 0.978 & $4.63^{j}$ & -0.54 & -0.32 \\
\hline \multirow[t]{3}{*}{ motion $^{j}$} & $49^{h}+3$ & 2OED & $\mathrm{H}^{\mathrm{N}}, \mathrm{H}^{\alpha}$ & 0.35 & 0.990 & $10.43^{j}$ & -1.12 & -0.46 \\
\hline & & & $\mathrm{H}^{\mathrm{N}}, \mathrm{C}^{\prime}$ & 0.33 & 0.927 & $5.44^{j}$ & -1.18 & -0.53 \\
\hline & & & $\mathrm{H}^{\mathrm{N}}, \mathrm{C}^{\beta}$ & 0.23 & 0.981 & $4.63^{j}$ & -0.60 & -0.37 \\
\hline \multirow[t]{3}{*}{ rigid } & $47+3$ & $\begin{array}{l}\text { averaged } \\
\text { ensemble }^{k}\end{array}$ & $\mathrm{H}^{\mathrm{N}}, \mathrm{H}^{\alpha}$ & 0.36 & 0.989 & 8.19 & -1.29 & 0.46 \\
\hline & & & $\mathrm{H}^{\mathrm{N}}, \mathrm{C}^{\prime}$ & 0.31 & 0.935 & 4.11 & -1.06 & 0.11 \\
\hline & & & $\mathrm{H}^{\mathrm{N}}, \mathrm{C}^{\beta}$ & 0.21 & 0.983 & 3.56 & -0.55 & 0.15 \\
\hline \multirow[t]{3}{*}{ motion $^{k}$} & $47+3$ & $\begin{array}{l}\text { averaged } \\
\text { ensemble }^{k}\end{array}$ & $\mathrm{H}^{\mathrm{N}}, \mathrm{H}^{\alpha}$ & 0.38 & 0.988 & 8.55 & -1.31 & 0.30 \\
\hline & & & $\mathrm{H}^{\mathrm{N}}, \mathrm{C}^{\prime}$ & 0.30 & 0.938 & 4.37 & -1.09 & 0.00 \\
\hline & & & $\mathrm{H}^{\mathrm{N}}, \mathrm{C}^{\beta}$ & 0.21 & 0.983 & 3.69 & -0.55 & 0.07 \\
\hline \multirow[t]{3}{*}{$\begin{array}{c}\text { indepen } \\
\text { dent }^{\prime}\end{array}$} & $47+3$ & ensemble & $\mathrm{H}^{\mathrm{N}}, \mathrm{H}^{\alpha}$ & 0.36 & 0.989 & 8.40 & -1.36 & 0.33 \\
\hline & & & $\mathrm{H}^{\mathrm{N}}, \mathrm{C}^{\prime}$ & 0.30 & 0.937 & 4.36 & -1.08 & -0.01 \\
\hline & & & $\mathrm{H}^{\mathrm{N}}, \mathrm{C}^{\beta}$ & 0.22 & 0.983 & 3.71 & -0.59 & 0.08 \\
\hline
\end{tabular}

${ }^{a}$ The following RDC restraints were used in NMR structure determination: $\mathrm{C}^{\alpha}-\mathrm{C}^{\prime}, \mathrm{C}^{\prime}-\mathrm{N}$ and $\mathrm{N}-\mathrm{H}^{\mathrm{N}}(1 \mathrm{P} 7 \mathrm{E}) ; \mathrm{C}^{\alpha}-\mathrm{C}^{\prime}, \mathrm{C}^{\prime}-\mathrm{N}$ and $\mathrm{C}^{\alpha}-\mathrm{H}^{\alpha}(1 \mathrm{P} 7 \mathrm{~F}) ; \mathrm{C}^{\alpha}-\mathrm{C}^{\prime}, \mathrm{C}^{\prime}-\mathrm{N}, \mathrm{C}^{\alpha}-\mathrm{H}^{\alpha}$ and $\mathrm{N}-\mathrm{H}^{\mathrm{N}}(2 \mathrm{OED}$ and ensemble).

b Pearson's correlation coefficient.

${ }^{\mathrm{c}}$ Convention used in equation 4 , main text.

$\mathrm{d}$ " +3 " refers to three ubiquitin residues with positive $\phi$ angles, A46, N60 and E64, included in the fit, using angles from an NMR-refined X-ray structure. ${ }^{5}$ Highly dynamic residues 12 and 40 are excluded in the fits.

${ }^{\mathrm{e}}$ Residues 10, 20, 21, 23, 24, 25, 32, 34, 36, 39 and 55, which have $>1.5$ standard deviation in the fits with $47+3$ residues, and residue 48 , which has $S^{2}<0.75$, were excluded in the fits.

${ }^{f}$ rmsd values of the phi angles from the structure reported in Bouvignies et al. ${ }^{6}$ are used for $\sigma$. Missing values and those of ubiquitin are set to the gamma amplitude (if available), and otherwise to $10^{\circ}$.

"Karplus coefficient " $A$ " is fixed in the fits to the value obtained for the $35+3$ "rigid" residues assuming $\sigma=10^{\circ}$.

${ }^{\mathrm{h}}$ Residues 12 and 40 are included.

"Karplus coefficient " $\mathrm{A}$ " is fixed in the fits to the value obtained for the $35+3$ "rigid" residues assuming $\sigma=0^{\circ}$.

j Karplus coefficient " $\mathrm{A}$ " is fixed in the fits to the value obtained for the $35+3$ "rigid" residues assuming $\sigma=20^{\circ}$. 
${ }^{k}$ Dihedral angles are calculated for each of the 160 ensemble conformers and subsequently averaged. "Rigid" refers to conventional SVD fitting of observed ${ }^{3} J$ couplings (all values except for dynamically disordered L12 and D40) to a static structure. "Motion" refers to the results obtained when using eq 5 , and rms devations from an averaged $\phi$ angle is used as $\sigma$.

${ }^{1}$ Karplus coefficients are derived from a fit to Karplus equations for each of the 160 ensemble conformers. Since the observed $J$ couplings are linear averages over the coupling of each conformer, the $N \times M$ linear equation system $(N=$ number of coupling types, $M=$ number of $\phi$ angles evaluated) to be minimized is given by:

$$
J^{\mathrm{ik}, \text { observed }}=\frac{1}{P} \sum_{l=1}^{P} J^{\mathrm{ikl}}=A_{\mathrm{k}}<\cos ^{2}\left(\phi_{\mathrm{il}}+\eta_{\mathrm{ikl}}\right)>+B_{\mathrm{k}}<\cos \left(\phi_{\mathrm{il}}+\eta_{\mathrm{ikl}}\right)>+C_{\mathrm{k}}
$$

where $\mathrm{i} \in\{1, \ldots, \mathrm{M}\}, \mathrm{k} \in\{1, \ldots, \mathrm{N}\}$ and 1 sums over $P$ ensemble conformers. 
Table S7. Root-mean-square $\phi$ angle related angular fluctuations in GB3 as derived from $\mathrm{RDC}$ and ${ }^{3} J$ analysis.

\begin{tabular}{|c|c|c|c|c|c|c|c|}
\hline & & $\mathrm{RDC}^{\mathrm{a}}$ & J Fits $49+3^{b}$ & J Fits $49+3^{c}$ & J Fits $49+3^{d}$ & J Fits $49+3^{\mathrm{e}}$ & ensemble \\
\hline Res\# & AA type & $\sigma_{\gamma}[$ degrees $]$ & $\sigma$ [degrees] & $\sigma$ [degrees] & $\sigma$ [degrees] & $\sigma$ [degrees] & $\begin{array}{c}\text { rmsd } \\
\text { [degrees] }\end{array}$ \\
\hline 3 & TYR & 0 & 9.8 & 12.2 & 20.6 & 14.5 & 8.4 \\
\hline 4 & LYS+ & 22 & 10.4 & 7.5 & 18.6 & 15.0 & 6.6 \\
\hline 5 & LEU & 0 & 11.2 & 16.1 & 22.2 & 15.8 & 4.8 \\
\hline 6 & VAL & 21 & 4.1 & 7.1 & 21.2 & 14.1 & 6.9 \\
\hline 7 & ILE & 0 & 9.8 & 12.1 & 21.0 & 14.2 & 9.0 \\
\hline 8 & ASN & 7 & 4.5 & 13.2 & 21.5 & 18.6 & 6.8 \\
\hline 9 & GLY & 2 & & & & & 12.0 \\
\hline 10 & LYS+ & 30 & 13.7 & 21.3 & 27.4 & 23.0 & 12.2 \\
\hline 11 & THR & 15 & 2.0 & 3.7 & 16.4 & 13.1 & 11.8 \\
\hline 12 & LEU & 33 & 24.2 & 29.4 & 35.6 & 30.0 & 17.8 \\
\hline 13 & LYS+ & 23 & 7.0 & 10.5 & 18.8 & 13.9 & 14.6 \\
\hline 14 & GLY & 26 & & & & & 15.7 \\
\hline 15 & GLU & 17 & 16.7 & 18.8 & 24.8 & 23.2 & 10.4 \\
\hline 16 & THR & 22 & 16.9 & 14.3 & 23.9 & 31.2 & 10.4 \\
\hline 17 & THR & 18 & 7.7 & 13.1 & 20.3 & 14.2 & 9.9 \\
\hline 18 & THR & 12 & 10.3 & 21.1 & 18.8 & 13.0 & 8.7 \\
\hline 19 & LYS+ & 17 & 13.2 & 12.8 & 25.9 & 20.5 & 10.1 \\
\hline 20 & ALA & 7 & 14.1 & 11.0 & 18.3 & 19.9 & 9.9 \\
\hline 21 & VAL & 0 & 7.1 & 19.5 & 30.1 & 53.7 & 10.3 \\
\hline 22 & ASP & 16 & 6.1 & 16.5 & 14.3 & 32.3 & 8.4 \\
\hline 23 & ALA & 11 & 3.6 & 15.1 & 17.8 & 23.9 & 4.4 \\
\hline 24 & GLU & 19 & 16.3 & 3.2 & 12.4 & 15.6 & 14.1 \\
\hline 25 & THR & & 6.0 & 0.7 & 17.1 & 9.7 & 6.2 \\
\hline 26 & ALA & 11 & 5.7 & 15.3 & 18.2 & 13.2 & 2.9 \\
\hline 28 & LYS+ & 9 & 13.1 & 16.8 & 21.1 & 24.7 & 2.1 \\
\hline 29 & ALA & 9 & 9.5 & 7.5 & 13.0 & 11.5 & 2.1 \\
\hline 30 & $\mathrm{PHE}$ & 11 & 8.7 & 12.5 & 21.2 & 11.0 & 2.0 \\
\hline 31 & LYS+ & 11 & 14.1 & 16.5 & 21.7 & 31.3 & 2.5 \\
\hline 32 & GLN & 10 & 14.5 & 9.9 & 28.4 & 14.6 & 3.2 \\
\hline 33 & TYR & 11 & 6.7 & 1.2 & 10.2 & 4.5 & 2.1 \\
\hline 34 & ALA & 18 & 13.8 & 5.4 & 15.2 & 9.8 & 3.8 \\
\hline 35 & ASN & 10 & 7.7 & 7.8 & 22.1 & 14.6 & 7.3 \\
\hline 36 & ASP & 11 & 13.2 & 23.2 & 33.4 & 26.4 & 4.7 \\
\hline 37 & ASN & 17 & 10.7 & 13.7 & 21.7 & 11.6 & 9.6 \\
\hline 38 & GLY & 17 & & & & & 11.9 \\
\hline 39 & VAL & 17 & 12.8 & 1.1 & 15.9 & 13.8 & 12.6 \\
\hline 40 & ASP & 0 & 16.7 & 21.2 & 30.7 & 31.6 & 15.8 \\
\hline 41 & GLY & 32 & & & & & 15.4 \\
\hline 42 & VAL & 10 & 14.7 & 11.8 & 24.3 & 21.3 & 9.1 \\
\hline 43 & TRP & 1 & 11.3 & 19.4 & 23.6 & 26.3 & 6.3 \\
\hline 44 & THR & 5 & 3.3 & 18.0 & 23.4 & 23.3 & 6.0 \\
\hline 45 & TYR & 17 & 20.2 & 17.0 & 26.0 & 13.0 & 11.1 \\
\hline 46 & ASP & 8 & 8.7 & 12.3 & 20.4 & 15.1 & 10.1 \\
\hline
\end{tabular}




\begin{tabular}{|c|c|c|c|c|c|c|c|}
\hline 47 & ASP & 4 & 10.1 & 16.9 & 33.7 & 25.4 & 9.4 \\
\hline 48 & ALA & 25 & 14.5 & 23.7 & 22.3 & 37.7 & 11.6 \\
\hline 49 & THR & 10 & 0.5 & 2.0 & 16.0 & 13.3 & 8.8 \\
\hline 50 & LYS+ & 17 & 14.7 & 21.3 & 29.0 & 24.1 & 3.9 \\
\hline 51 & THR & 0 & 1.4 & 1.9 & 18.0 & 10.0 & 8.1 \\
\hline 52 & PHE & 0 & 6.8 & 12.6 & 20.6 & 15.8 & 5.6 \\
\hline 53 & THR & 24 & 6.9 & 11.6 & 20.7 & 10.9 & 8.2 \\
\hline 54 & VAL & 0 & 6.6 & 7.8 & 18.2 & 12.9 & 4.2 \\
\hline 55 & THR & 21 & 10.7 & 11.0 & 18.9 & 14.0 & 10.9 \\
\hline 56 & GLU & 18 & 4.3 & 14.6 & 24.4 & 28.1 & 16.8 \\
\hline \multicolumn{2}{|c|}{ average } & 13.4 & 10.1 & 12.9 & 21.6 & 19.2 & 8.6 \\
\hline \multicolumn{2}{|c|}{ standard dev. } & 9.3 & 5.0 & 6.5 & 5.5 & 8.9 & 4.1 \\
\hline \multicolumn{8}{|c|}{ Ubiquitin } \\
\hline 46 & ALA & & 10.6 & 17.5 & 24.1 & 22.5 & \\
\hline 60 & ASN & & 7.6 & 21.0 & 23.9 & 17.5 & \\
\hline 64 & GLU & & 17.9 & 21.6 & 23.9 & 22.3 & \\
\hline
\end{tabular}

${ }^{\text {a }}$ From Bouvignies et al. ${ }^{6}$

${ }^{\mathrm{b}}$ From fits of the ${ }^{3} \mathrm{~J}$ couplings to $2 \mathrm{OED}$, using eq 5 , and keeping coefficients $A$ fixed at the values reported in Table S6, for the set of " $35+3$ " residues assuming $\sigma=0$.

${ }^{\mathrm{c}}$ From fits of the ${ }^{3} \mathrm{~J}$ couplings to $2 \mathrm{OED}$, using eq 5 , and keeping coefficients $A$ fixed at the values reported in Table S6, for the set of “ $35+3$ " residues assuming $\sigma=10^{\circ}$.

${ }^{\mathrm{d}}$ From fits of the ${ }^{3} \mathrm{~J}$ couplings to $2 \mathrm{OED}$, using eq 5 , and keeping coefficients $A$ fixed at the values reported in Table S6, for the set of " $35+3$ " residues assuming $\sigma=20^{\circ}$.

${ }^{\mathrm{e}}$ From fits of the ${ }^{3} \mathrm{~J}$ couplings to $1 \mathrm{IGD}$, using eq 5, and keeping coefficients $A$ fixed at the values reported in Table S6, for the set of "35+3" residues assuming $\sigma=10^{\circ}$. 
Table S8. ${ }^{3} J_{\mathrm{HN}, \mathrm{H} \alpha}$ Karplus coefficients from different parameterizations.

\begin{tabular}{|c|c|c|c|c|}
\hline $\begin{array}{l}\text { fitting } \\
\text { model }^{b}\end{array}$ & $A[\mathrm{~Hz}]$ & $B[\mathrm{~Hz}]$ & $C[\mathrm{~Hz}]$ & reference \\
\hline rigid & 8.0 & -0.9 & 0.9 & $\begin{array}{l}\text { Bystrov, V.F., Portnova, S.L., Tsetlin, V.L., Ivanov, V.T. } \\
\text { and Ovchinnikov, Y.A., 1969, Tetrahedron, 25, } 493\end{array}$ \\
\hline rigid & 7.9 & -1.55 & 1.35 & $\begin{array}{l}\text { Ramachandran, G.N., Chandrasekaran, R. and } \\
\text { Kopple, K.D., 1971, Biopolymers, 10, } 2113\end{array}$ \\
\hline rigid & 9.4 & -1.1 & 0.4 & $\begin{array}{c}\text { Bystrov, V.F., Ivanov, V.T., Portnova, S.L., Balashova, } \\
\text { T.A. and Ouchinnikov, Y.A., 1973, Tetrahedron, 29, } \\
873\end{array}$ \\
\hline rigid & 5.4 & -1.3 & 2.2 & $\begin{array}{l}\text { DeMarco, A., Llinas, M. and Wuthrich, K., 1978, } \\
\text { Biopolymers, 17, } 637\end{array}$ \\
\hline rigid & 6.4 & -1.4 & 1.9 & $\begin{array}{c}\text { Pardi, A., Billeter, M. and Wuthrich, K., 1984, J. Mol. } \\
\text { Biol., 180,, 741-751 }\end{array}$ \\
\hline rigid & 6.7 & -1.3 & 1.5 & $\begin{array}{l}\text { Ludvigsen, S., Andersen, K.V., Poulsen, F.M., 1991, J. } \\
\text { Mol. Biol., 217, 731-736 }\end{array}$ \\
\hline rigid & 6.0 & -1.4 & 2.4 & $\begin{array}{l}\text { Smith, L.J., Sutcliffe, M.J., Redfield, C. and Dobson, } \\
\text { C.M., 1991, Biochemistry, 30, } 986\end{array}$ \\
\hline rigid & 6.51 & -1.76 & 1.60 & $\begin{array}{c}\text { Vuister,G.W. and Bax, A.,1993, J. Am. Chem. Soc., } \\
\text { 115, 7772-7777 }\end{array}$ \\
\hline rigid & 6.98 & -1.38 & 1.72 & $\begin{array}{l}\text { Wang, A.C. and Bax. A., 1996, J. Am. Chem. Soc., } \\
118,2483-2494\end{array}$ \\
\hline rigid & 7.90 & -1.05 & 0.65 & $\begin{array}{c}\text { Schmidt, J.M., Blumel, M., Lohr, F. and Ruterjans, H., } \\
\text { 1999, J. Biomol. NMR, 14, 1-12 }\end{array}$ \\
\hline rigid & 7.13 & $|\mathrm{~B}|=0.87$ & 0.10 & $\begin{array}{c}\text { Habeck, M., Rieping, W. and Nilges, M., 2005, J. } \\
\text { Magn. Reson., 177, 160-165 }\end{array}$ \\
\hline rigid & 7.97 & -1.26 & 0.63 & Current work, using 2OED as a reference \\
\hline $\begin{array}{l}\text { DFT(Ace- } \\
\text { Ala-NMe) }\end{array}$ & 9.44 & -1.53 & -0.07 & $\begin{array}{c}\text { Case, D.A., Scheurer, C. and Bruschweiler, J. Am. } \\
\text { 2000, Chem. Soc., 116, 11199-11200 R., }\end{array}$ \\
\hline $\begin{array}{l}\text { DFT(Ala- } \\
\text { Ala-NH }\end{array}$ & 9.14 & -2.28 & -0.29 & $\begin{array}{l}\text { Case, D.A., Scheurer, C. and Bruschweiler, J. Am. } \\
\text { 2000, Chem. Soc., 116, 11199-11200 R., }\end{array}$ \\
\hline motion & 9.5 & -1.4 & 0.3 & $\begin{array}{l}\text { Bruschweiler, R. and Case, D.A.,1994, J. Am. Chem. } \\
\text { Soc., 116, 11199-11200 }\end{array}$ \\
\hline motion & 8.69 & -1.14 & 0.39 & $\begin{array}{c}\text { Current work, using 2OED as a reference, and } \\
\text { assuming }\langle\sigma\rangle=10^{\circ} \text { for " } 35+3^{\text {" well-defined residues }}\end{array}$ \\
\hline ensemble & 8.40 & -1.36 & 0.33 & $\begin{array}{c}\text { Current work, using the } 160 \text { member ensemble of } \\
\text { Clore, G. M.and Schwieters, C. D., } 2006 \text { J. Mol. Biol., } \\
\text { 355, 879-886 }\end{array}$ \\
\hline
\end{tabular}


Table S9. ${ }^{3} J_{\mathrm{HN}, \mathrm{C}^{\prime}}$ Karplus coefficients from different parameterizations.

\begin{tabular}{|c|c|c|c|c|}
\hline $\begin{array}{l}\text { fitting } \\
\text { model }^{b}\end{array}$ & $A[\mathrm{~Hz}]$ & $B[\mathrm{~Hz}]$ & $C[\mathrm{~Hz}]$ & reference \\
\hline rigid & 5.7 & -2.7 & 0.1 & $\begin{array}{c}\text { Seip, S., Balbach, J. and Kessler, H., 1994, J. Magn. } \\
\text { Reson. B, 104, 172-179 }\end{array}$ \\
\hline rigid & 5.8 & -2.7 & 0.1 & $\begin{array}{c}\text { Weisemann, R.,Ruterjans, H., Schwalbe, H., } \\
\text { Schleucher, J., Bermel, W. And Griesinger, C., 1994, } \\
\text { J. Biomol. NMR, 4, 231-240, origin unclear }\end{array}$ \\
\hline rigid & 4.0 & -1.1 & 0.1 & $\begin{array}{l}\text { Wang, A.C. and Bax, A., 1995, J. Am. Chem. Soc., } \\
\text { 117, 1810-1813 }\end{array}$ \\
\hline rigid & 4.32 & -0.84 & 0.00 & $\begin{array}{l}\text { Wang, A.C. and Bax. A., 1996, J. Am. Chem. Soc., } \\
118,2483-2494\end{array}$ \\
\hline rigid & 4.41 & -1.36 & 0.24 & $\begin{array}{c}\text { Schmidt, J.M., Blumel, M., Lohr, F. and Ruterjans, H., } \\
\text { 1999, J. Biomol. NMR, 14, 1-12 }\end{array}$ \\
\hline rigid & 4.19 & $|\mathrm{~B}|=0.99$ & 0.03 & $\begin{array}{l}\text { Habeck, M., Rieping, W. and Nilges, M., 2005, J. } \\
\text { Magn. Reson., 177, 160-165 }\end{array}$ \\
\hline rigid & 4.12 & -1.10 & 0.11 & Current work, using 2OED as a reference \\
\hline $\begin{array}{l}\text { DFT(Ace- } \\
\text { Ala-NMe) }\end{array}$ & 5.58 & -1.06 & -0.30 & $\begin{array}{c}\text { Case, D.A., Scheurer, C. and Bruschweiler, J. Am. } \\
\text { 2000, Chem. Soc., 116, 11199-11200 R. }\end{array}$ \\
\hline $\begin{array}{l}\text { DFT(Ala- } \\
\text { Ala-NH }{ }_{2} \text { ) }\end{array}$ & 5.34 & -1.46 & -0.29 & $\begin{array}{l}\text { Case, D.A., Scheurer, C. and Bruschweiler, J. Am. } \\
\text { 2000, Chem. Soc., 116, 11199-11200 R., }\end{array}$ \\
\hline motion & 4.53 & -1.09 & -0.09 & $\begin{array}{c}\text { Current work, using 2OED as a reference, and } \\
\text { assuming }\langle\sigma\rangle=10^{\circ} \text { for " } 35+3 \text { " well-defined residues }\end{array}$ \\
\hline ensemble & 4.36 & -1.08 & -0.01 & $\begin{array}{c}\text { Current work, using the } 160 \text { member ensemble of } \\
\text { Clore, G. M.and Schwieters, C. D., } 2006 \text { J. Mol. Biol., } \\
355,879-886\end{array}$ \\
\hline
\end{tabular}

Table S10. ${ }^{3} J_{\mathrm{HN}, \mathrm{C \beta}}$ Karplus coefficients from different parameterizations.

\begin{tabular}{|c|c|c|c|c|}
\hline $\begin{array}{l}\text { fitting } \\
\text { model }^{b}\end{array}$ & $A[\mathrm{~Hz}]$ & $B[\mathrm{~Hz}]$ & $C[\mathrm{~Hz}]$ & reference \\
\hline rigid & 4.5 & -1.5 & -0.2 & $\begin{array}{c}\text { Seip, S., Balbach, J. and Kessler, H., 1994, J. Magn. } \\
\text { Reson. B, 104, 172-179 }\end{array}$ \\
\hline rigid & 3.39 & -0.94 & 0.07 & $\begin{array}{l}\text { Wang, A.C. and Bax. A., 1996, J. Am. Chem. Soc., } \\
118,2483-2494\end{array}$ \\
\hline rigid & 2.90 & -0.56 & 0.18 & $\begin{array}{c}\text { Schmidt, J.M., Blumel, M., Lohr, F. and Ruterjans, H., } \\
\text { 1999, J. Biomol. NMR, 14, 1-12 }\end{array}$ \\
\hline rigid & 3.26 & $|B|=0.87$ & 0.10 & $\begin{array}{c}\text { Habeck, M., Rieping, W. and Nilges, M., 2005, J. } \\
\text { Magn. Reson., 177, 160-165 }\end{array}$ \\
\hline rigid & 3. 51 & -0.53 & 0.14 & Current work, using 2OED as a reference \\
\hline $\begin{array}{l}\text { DFT(Ace- } \\
\text { Ala-NMe) }\end{array}$ & 5.15 & 0.01 & -0.32 & $\begin{array}{c}\text { Case, D.A., Scheurer, C. and Bruschweiler, J. Am. } \\
\text { 2000, Chem. Soc., 116, 11199-11200 R. }\end{array}$ \\
\hline $\begin{array}{l}\text { DFT(Ala- } \\
\text { Ala-NH }{ }_{2} \text { ) }\end{array}$ & 4.58 & -0.36 & -0.31 & $\begin{array}{c}\text { Case, D.A., Scheurer, C. and Bruschweiler, J. Am. } \\
\text { 2000, Chem. Soc., 116, 11199-11200 R., }\end{array}$ \\
\hline motion & 3.85 & -0.47 & -0.08 & $\begin{array}{l}\text { Current work, using 2OED as a reference, and } \\
\text { assuming }\langle\sigma\rangle=10^{\circ} \text { for " } 35+3 \text { " well-defined residues }\end{array}$ \\
\hline ensemble & 3.71 & -0.59 & 0.08 & $\begin{array}{l}\text { Current work, using the ensemble of Clore, G. M.and } \\
\text { Schwieters, C. D., } 2006 \text { J. Mol. Biol., 355, 879-886 }\end{array}$ \\
\hline
\end{tabular}




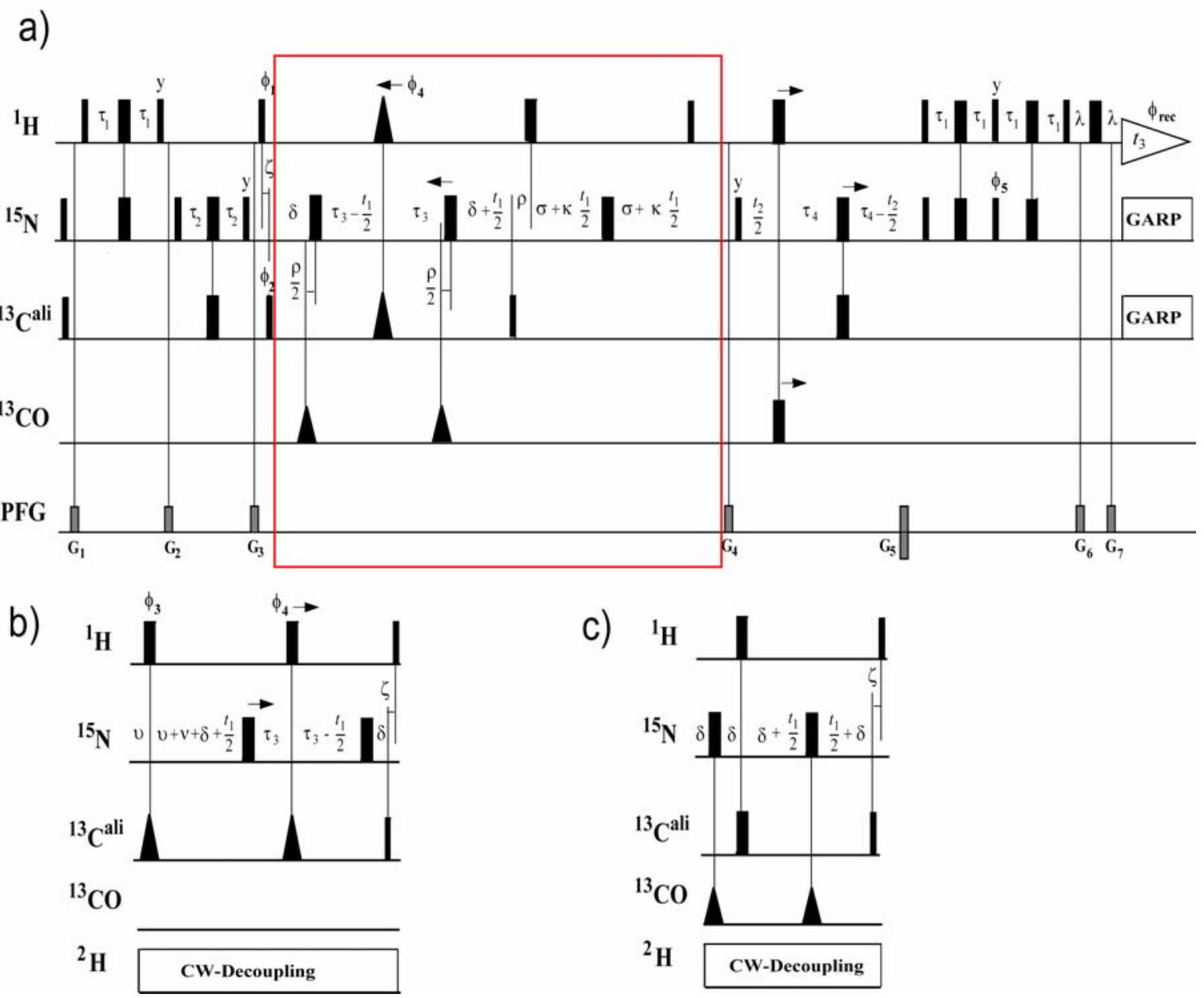

Figure S4. Pulse diagrams of the schemes shown in Figure 1, main text, but including small compensation delays $(\delta, v, v, \sigma, \rho, \zeta)$ and Bloch-Siegert pulses, used to ensure the absence of frequency-dependent phase errors in the indirectly detected dimensions. The radio-frequency pulses on ${ }^{1} \mathrm{H},{ }^{15} \mathrm{~N},{ }^{13} \mathrm{C}^{\alpha}$ and ${ }^{13} \mathrm{C}$ ' are applied at $4.7,120,56$ and $174 \mathrm{ppm}$, respectively. Narrow and wide bars indicate non-selective $90^{\circ}$ and $180^{\circ}$ pulses. Triangular pulses on ${ }^{13} \mathrm{C}^{\prime}$ represent selective $180^{\circ}$-Gaussian pulses of length $p_{\mathrm{C}^{\prime}}{ }^{180}=150 \mu \mathrm{s}$, triangular pulses on ${ }^{13} \mathrm{C}^{\text {aliphatic }}$ in (a) represent $180^{\circ}$-REBURP pulses of length $p_{\text {Cali }}{ }^{180}=$ $400 \mu$ s with a full-inversion range of $\pm 33 \mathrm{ppm}$ at $151 \mathrm{MHz}{ }^{13} \mathrm{C}$ frequency, centered at 50 ppm, and in (b) hyperbolic secant pulses of length $p_{\text {Cali }}{ }^{180}=700 \mu$ s with a full-inversion range of $\pm 80 \mathrm{ppm}$; the triangular pulse on ${ }^{1} \mathrm{H}$ is a $\mathrm{H}^{\mathrm{N}}$-selective REBURP pulse of length $p_{\mathrm{H}}{ }^{180}=1.5 \mathrm{~ms}$ with a full-inversion range of $\pm 2.3 \mathrm{ppm}$ applied at $8.87 \mathrm{ppm}$. Vertical lines connect centered pulses. ${ }^{15} \mathrm{~N}$ - and ${ }^{13} \mathrm{C}^{\text {ali }}$-decoupling is achieved using GARP modulation at RF field strengths, $\gamma B_{1}$, of $1.1 \mathrm{kHz}$ and $2.08 \mathrm{kHz}$, and ${ }^{2} \mathrm{H}$-decoupling with continuouswave irradiation using $\gamma B_{1} \approx 700 \mathrm{~Hz}$ (also applied during the $\mathrm{N}-\mathrm{C}^{\alpha}$ and $\mathrm{C}^{\alpha}-\mathrm{N}$ transfers). Unless indicated otherwise, all RF pulses are applied with phase $\mathrm{x}$. Phase cycling: $\phi_{3}=$ $\{\mathrm{x}, \mathrm{x}, \mathrm{x}, \mathrm{x},-\mathrm{x},-\mathrm{x},-\mathrm{x},-\mathrm{x}\} ; \phi_{4}=\{\mathrm{x},-\mathrm{x}, \mathrm{x},-\mathrm{x}\} ; \phi_{5}=\mathrm{y} ; \phi_{\mathrm{rec}}=\{\mathrm{x},-\mathrm{x},-\mathrm{x}, \mathrm{x}\}$. For spectrum $\mathrm{A}: \phi_{1}=$ $\{\mathrm{x}, \mathrm{x},-\mathrm{x},-\mathrm{x}\} ; \phi_{2}=\{\mathrm{x},-\mathrm{x}, \mathrm{x},-\mathrm{x}\}$. For spectrum B: $\phi_{1}=\{\mathrm{y}, \mathrm{y},-\mathrm{y},-\mathrm{y}\} ; \phi_{2}=\{\mathrm{y},-\mathrm{y}, \mathrm{y},-\mathrm{y}\} . \mathrm{A}-\mathrm{B}$ $(A+B)$ selects DQ (ZQ) components. The delays have the following values: $\tau_{1}=1 /\left(4 J_{\mathrm{H}, \mathrm{N}}\right)$ $=2.6 \mathrm{~ms}, \tau_{2}=1 /\left(4 J_{\mathrm{N}, \mathrm{C} \alpha}\right)=13.7 \mathrm{~ms}, \tau_{3}=1 /\left(2 J_{\mathrm{C} \alpha, \mathrm{C} \beta}\right)-\delta-p_{\mathrm{C}}{ }^{180}-\mathrm{d}-4 p_{\mathrm{Cali}}{ }^{90} / \pi=13 \mathrm{~ms}$ 
(where $p_{\text {Cali }}{ }^{90}$ is the length of a $90^{\circ}$-pulse on ${ }^{13} \mathrm{C}^{\alpha}$ and $\mathrm{d}$ is the effective $J_{\mathrm{C} \alpha, \mathrm{C} \beta}$-coupling time during the ${ }^{13} \mathrm{C}^{\text {ali }}$ - and ${ }^{1} \mathrm{H}-180^{\circ}$ pulses; in experiment $\mathrm{b},{p_{\mathrm{C}}}^{180}$ is replaced by $p_{\mathrm{N}}{ }^{180}$ ) or $27 \mathrm{~ms}$ in $\mathrm{b}, \tau_{4}=1 /\left(4 J_{\mathrm{N}, \mathrm{C} \alpha}\right)=13.5 \mathrm{~ms}, \delta=13 \mu \mathrm{s}, \mathrm{v}=4 \mu \mathrm{s}$ (required for change of power level on the ${ }^{13} \mathrm{C}^{\text {ali }}$ channel), $v=4 p_{\text {Cali }}{ }^{90} / \pi$ for refocusing ${ }^{13} \mathrm{C}$-chemical-shift evolution and $J_{\mathrm{C} \alpha, \mathrm{C}}$, and $J_{\mathrm{C} \alpha, \mathrm{H} \alpha}$ coupling, $\zeta=2\left(p_{\mathrm{H}}{ }^{90}-p_{\mathrm{Cali}}{ }^{90}\right) / \pi$ (where $p_{\mathrm{H}}{ }^{90}$ is the length of a $90^{\circ}$-pulse on ${ }^{1} \mathrm{H}$ ) for refocusing ${ }^{1} \mathrm{H}^{\mathrm{N}}$-chemical-shift evolution and $J_{\mathrm{HN}, \mathrm{H \alpha}}$-coupling, $\lambda=320 \mu \mathrm{s}, \rho=$ $4 p_{\mathrm{H}}{ }^{90} / \pi+2 \sigma+p_{\mathrm{N}}{ }^{180}+\zeta$ is adjusted for exact refocusing of the ${ }^{1} \mathrm{H}^{\mathrm{N}}$-chemical-shift evolution and $J_{\mathrm{H}, \mathrm{N}}$-coupling during the total MQ- and SQ-period for $t_{1}=0, \sigma=10 \mu \mathrm{s}$. Pulsed field gradients indicated on the line marked PFG are applied along the $\mathrm{z}$ axis $\left(\mathrm{G}_{1-}\right.$ 4) or along xyz $\left(\mathrm{G}_{5-7}\right)$ with duration and strength of: $\mathrm{G}_{1}, 500 \mu \mathrm{s}, 18 \mathrm{G} / \mathrm{cm} ; \mathrm{G}_{2}, 300 \mu \mathrm{s}, 36$ $\mathrm{G} / \mathrm{cm} ; \mathrm{G}_{3}, 500 \mu \mathrm{s}, 18 \mathrm{G} / \mathrm{cm} ; \mathrm{G}_{4}, 250 \mu \mathrm{s}, 15 \mathrm{G} / \mathrm{cm} ; \mathrm{G}_{5}, 3400 \mu \mathrm{s},-30 \mathrm{G} / \mathrm{cm} ; \mathrm{G}_{6}, 144.75 \mu \mathrm{s}$, $-30 \mathrm{G} / \mathrm{cm} ; \mathrm{G}_{7}, 200 \mu \mathrm{s}, 30 \mathrm{G} / \mathrm{cm}$. Quadrature detection in the $\mathrm{MQ}\left(t_{1}\right)$ and ${ }^{15} \mathrm{~N}\left(t_{2}\right)$ dimension is achieved by the States-TPPI method applied simultaneously to the phases $\phi_{1}$ and $\phi_{2}$, and by the echo-antiecho method, applied by inversion of $\mathrm{G}_{5}$ and $\phi_{5}$, respectively. Pulse sequence code for Bruker spectrometers, acquisition parameters, and NMRPipe processing scripts can be downloaded from http://spin.niddk.nih.gov/bax/main.html. 

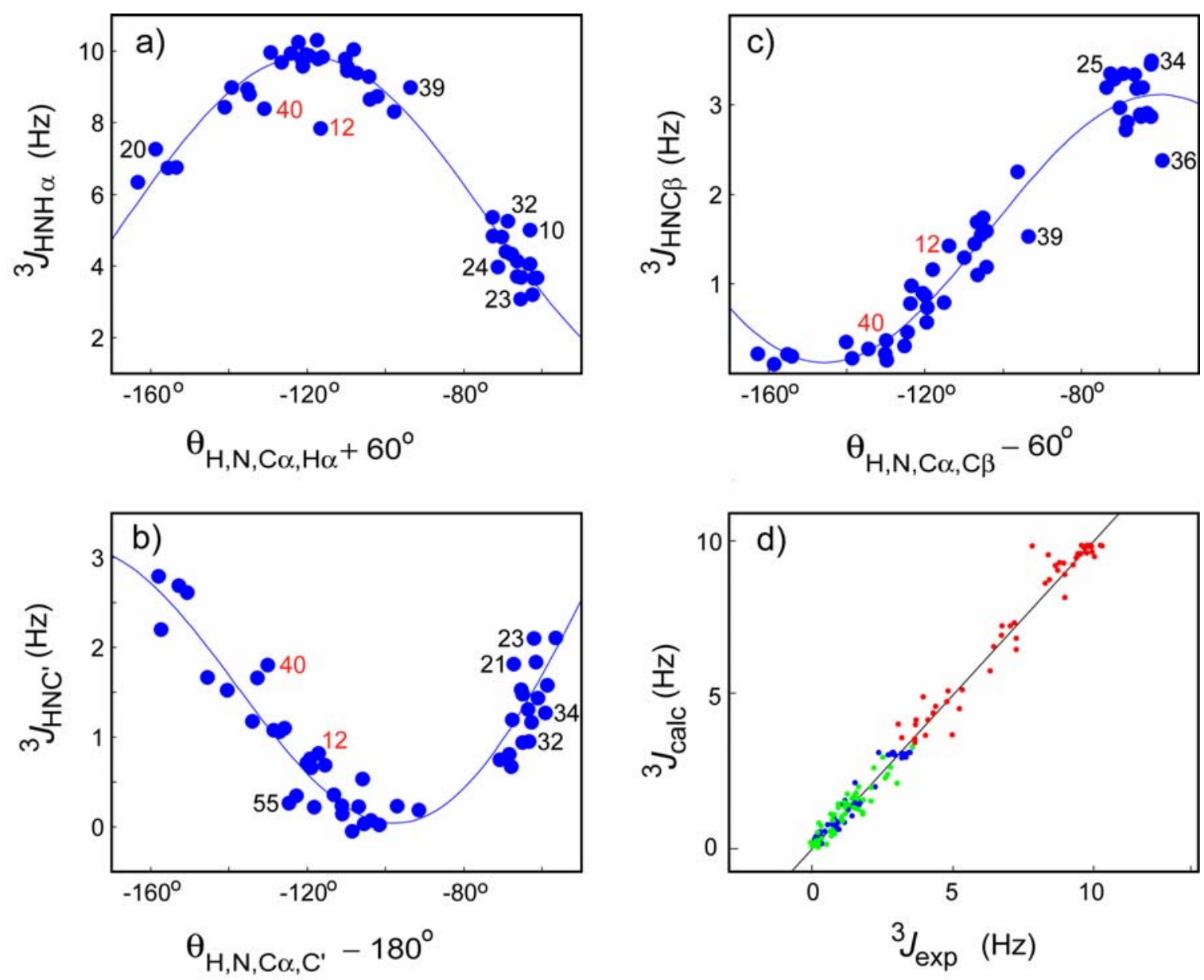

Figure S5. ${ }^{3} J_{\mathrm{HN}, \mathrm{H \alpha}},{ }^{3} J_{\mathrm{HN}, \mathrm{C}^{\prime}}$ and ${ }^{3} J_{\mathrm{HN}, \mathrm{C} \beta}$ versus their respective dihedral angles in the NMR structure, offset by $\pi / 3, \pi$, and $-\pi / 3$ for easy comparison of ${ }^{3} J$ values, observed for any given residue. Karplus curves shown are derived using singular value decomposition and a static protein structural model (2OED). L12 and D40, previously identified as highly dynamic on the basis of ${ }^{15} \mathrm{~N}$ relaxation, are marked in red; residues that deviate more than 1.5 standard deviations from the Karplus curve are marked in black. Marked residues were excluded when fitting the remaining residues to a dynamic Karplus curve with the assumption $\sigma=10^{\circ}$. (d) Plot of experimental ${ }^{3} \mathrm{~J}$ values versus values predicted by the static Karplus curve, using dihedral angles taken from the NMR structure, 2OED (red- ${ }^{3} J_{\mathrm{HN}, \mathrm{H} \alpha}$; green- ${ }^{3} J_{\mathrm{HN}, \mathrm{C}}$; blue-- ${ }^{3} J_{\mathrm{HN}, \mathrm{C} \beta}$ ). 


\section{References:}

(1) Frisch, M. J.; Trucks, G. W.; Schlegel, H. B.; Scuseria, G. E.; Robb, M. A.;

Cheeseman, J. R.; Montgomery, J., J. A.; Vreven, T.; Kudin, K. N.; Burant, J. C.; Millam, J. M.; Iyengar, S. S.; Tomasi, J.; Barone, V.; Mennucci, B.; Cossi, M.; Scalmani, G.; Rega, N.; Petersson, G. A.; Nakatsuji, H.; Hada, M.; Ehara, M.; Toyota, K.; Fukuda, R.; Hasegawa, J.; Ishida, M.; Nakajima, T.; Honda, Y.; Kitao, O.; Nakai, H.; Klene, M.; Li, X.; Knox, J. E.; Hratchian, H. P.; Cross, J. B.; Bakken, V.; Adamo, C.; Jaramillo, J.; Gomperts, R.; Stratmann, R. E.; Yazyev, O.; Austin, A. J.; Cammi, R.; Pomelli, C.; Ochterski, J. W.; Ayala, P. Y.; Morokuma, K.; Voth, G. A.; Salvador, P.; Dannenberg, J. J.; Zakrzewski, V. G.; Dapprich, S.; Daniels, A. D.; Strain, M. C.; Farkas, O.; Malick, D. K.; Rabuck, A. D.; Raghavachari, K.; Foresman, J. B.; Ortiz, J. V.; Cui, Q.; Baboul, A. G.; Clifford, S.; Cioslowski, J.; Stefanov, B. B.; Liu, G.; Liashenko, A.; Piskorz, P.; Komaromi, I.; Martin, R. L.; Fox, D. J.; Keith, T.; Al-Laham, M. A.; Peng, C. Y.; Nanayakkara, A.; Challacombe, M.; Gill, P. M. W.; Johnson, B.; Chen, W.; Wong, M. W.; Gonzalez, C.; Pople, J. A. Gaussian 03, Revision C.02, Gaussian Inc., Wallingford CT., 2004.

(2) Becke, A. D., J. Chem. Phys. 1993, 98, 5648-5652.

(3) Helgaker, T.; Watson, M.; Handy, N. C., J. Chem. Phys. 2000, 113, 9402-9409.

(4) Sychrovsky, V.; Grafenstein, J.; Cremer, D., J. Chem. Phys. 2000, 113, 35303547 .

(5) Hu, J. S.; Bax, A., J. Am. Chem. Soc. 1997, 119, 6360-6368.

(6) Bouvignies, G.; Bernado, P.; Meier, S.; Cho, K.; Grzesiek, S.; Bruschweiler, R.; Blackledge, M., Proc. Natl. Acad. Sci. U. S. A. 2005, 102, 13885-13890. 